\title{
Butterflies of Kedarnath Musk Deer Reserve, Garhwal Himalaya, India
}

\author{
Arun P. Singh \\ Entomology Division, Forest Research Institute, P.O. New Forest, Dehradun, Uttarakhand 248006, India \\ Email: singhap@icfre.org; ranoteaps@gmail.com
}

\begin{abstract}
A checklist of 147 species of butterflies recorded from Kedarnath Musk Deer Reserve in Chamoli and Rudraprayag districts of Uttarakhand state in India, studied during May 2006 - September 2008 is given along with their seasonality, altitudinal distribution and relative abundance in the study area.
\end{abstract}

Keywords: Broad leaved forest, butterflies, moist temperate, seasonality, Western Himalaya

Date of online publication 26 January 2009 ISSN 0974-7907 (online) | 0974-7893 (print)

Editor: Peter Smetacek

\section{Manuscript details:}

Ms \# 01873

Received 15 October 2007

Final revised received 30 September 2008

Finally accepted 09 October 2008

Citation: Singh, A.P. (2009). Butterflies of Kedarnath Musk Deer Reserve, Garhwal Himalaya, India. Journal of Threatened Taxa 1(1): $37-48$

Copyright: () Arun P. Singh 2009. Creative Commons Attribution 3.0 Unported License. JoTT allows unrestricted use of this article in any medium for non-profit purposes, reproduction and distribution by providing adequate credit to the authors and the source of publication.

Author Details: ARUN P. SINGH works on the conservation of biodiversity of the western Himalaya with special reference to butterflies and birds since past 15 years. His research work include ecology, taxonomy, environmental impact assessment (EIA) studies, along with teaching (Entomology and Wildlife Management) at Forest Research Institute University at Dehradun.

Acknowledgements: The present study is part of a research project (FRI-348/FED-23) of Forest Research Institute (FRI), Dehradun, India, being funded by the Indian Council of Forestry Research and Education (ICFRE). The author is thankful to Director, FRI and Head, Entomology Division, FRI, for providing the necessary facilities to carry out this study. Author is also thankful to Shri. B.C.Pandey and Shri. R. Kumar, Technical Assistants (FRI), for their help in collection of insect material from the field.
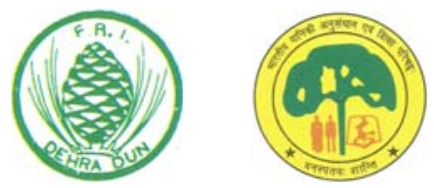

OPEN AGGESS | FREE DOWNLOAD

\section{INTRODUCTION}

Kedarnath Musk Deer Reserve (KMDR) was created in 1972 and is situated $300 \mathrm{~km}$ north of Delhi, covering an area of $975 \mathrm{~km}^{2}$. This sanctuary lies in the catchment of Alaknanda river, which is a major tributary of the upper reaches of the Ganga. It is bounded to the north by a range of peaks i.e. Chaukhamba $(7,068 \mathrm{~m})$ and Kedarnath (above 6,000m) and in the south by Mandal-Okhimath road varying from 1,100-2,870m (Fig. 1). The climate in the Reserve is considerably influenced by south west-monsoon in summer. Of the mean annual precipitation of $2,665 \mathrm{~mm}$ and $3,093 \mathrm{~mm}$ at $1,400 \mathrm{~m}$ and $3,053 \mathrm{~m}$, respectively, in the main study area (Fig. 1), $80 \%$ falls during June and September and $11 \%$ as snow between December and March. Temperatures are highest in May-June before the arrival of monsoon, and are lowest in the first week of January. The highest and lowest temperatures recorded at $3,050 \mathrm{~m}$ were $25.0^{\circ} \mathrm{C}$ and $-10^{\circ} \mathrm{C}$, respectively. The Reserve is snow bound for three months in the year, following heavy snowfall in December (Green 1986; Pande et al. 2001).

A great variety of vegetation types occur in the reserve, reflecting the complex and diverse climate, geology and topography of the region. The forests here stretch over the mountain slopes in large, dense and continuous patches with closed canopy. Besides, high rainfall, lichens on oak trees, dwarf bamboo, numerous fresh water streams and high plant diversity with more than 650 plant species (Semwal \& Gaur 1981; Pande et al. 2001; Singh et al. 2009) are the characteristics of the study area. Interestingly, the distribution of at least seven of 252 bird species found in the study area is known only from much further east in central Nepal (Myers \& Singh 2006; Singh \& Singh 2007). The sub-tropical, moist-temperate, sub-alpine and alpine zones in the reserve are reflected along an altitudinal gradient, while the tropical zone is absent, as per the classification of Champion \& Seth (1968). The sub-tropical zone consists of mixed 'broad-leaved' forest with Ban Oak (Quercus leucotrichophora) and Moru Oak (Q. dilatata) (1,500-2,750m), with Rhododendron arboreum often constituting a second under storey. Below these lie impenetrable thickets of Bamboo (Thamnocalamus sp.), Elm (Ulmus wallichiana), Horse Chestnut (Aesculus indica), Bird Cherry (Prunus padus), Hazel (Corylus colurna), Maple (Acer sp.) which are absent from the lower altitudes, are often associated with moru oak. The temperate zone consists of Karsu Oak (Q. semecarpifolia) forest (2,500-3,300m) along with Maple (Acer caecium), Meliosma dilleniaefolia, Rowan (Sorbus lanata), Yew (Taxus baccata) along with thickets of a Bamboo (T. spathiflorus) and conifer forest with mainly West Himalayan Silver Fir, Abies pindrow (2,600-3,400m) and Rhododendron arboreum. The Sub-alpine zone consists of Birch (Betula utilis), Dwarf Rhododendron (R. campanulatum), Scrub Forest (3,100-3,350m) intermingled with dense clumps of bamboo (T. spathiflorus). The Alpine zone (3,350-3,500m) consists of $R$. campanulatum scrub above which lie the 'pastures' (above $3,500 \mathrm{~m}$ ) that are dominated by the herb community Danthonia cumminsii, shrubs like Rhododendron lepidotum, R. barbatum and forbs Bistorta sp. (Green 1986).

Butterflies of KMDR have not been previously documented. Published literature from Garhwal Himalaya pertains mainly to areas lying south of KMDR, Dehradun and Tehri Garhwal districts (Mackinnon \& De Nicéville 1899; Ollenbach 1930; Evans 1932; Singh \& Bhandari 2003) and from adjoining areas of Kumaon Himalaya, Almora and Nainital districts (Doherty 1886; Hannyngton 1910). Besides, 35 species have been 
recorded from Nanda Devi National Park (Fig. 1), lying in the Garhwal Himalaya bordering Kumaon (Baindur 1993; Uniyal 2004).

\section{Materials and Methods}

The study area lies in the south-eastern part of KMDR between $30^{\circ} 27^{\prime} 72^{\prime \prime}-30^{\circ} 29^{\prime} 29^{\prime \prime} \mathrm{N} \& 79^{\circ} 08^{\prime} 82^{\prime \prime}-79^{\circ} 17^{\prime} 90^{\prime \prime} \mathrm{E}$ (Fig. 1). Butterflies were sampled from 13 May 2006 to 6 September 2008 using Pollard walk (Pollard et al. 1975; Walpole \& Sheldon 1999). Besides photographing butterflies, voucher specimens were also collected of species that could not be identified in the field. Ten transects of $2 \mathrm{~km}$ each were trekked, for $1.5 \mathrm{~h}$ each, on forest trails and Mandal - Okhimath road for sampling butterflies, which was done once in two months on the following trails/routes: Mandal (1,528m) - Anusuyia Devi Temple (2,100m), Mandal - Kanchula Kharak (2,660m), Kanchula Kharak - Chopta (2,870m), Chopta - Tunganth Temple (3,486m) - Chandrashila Peak (3,621m), Maku bend (2,250m) - Maku Math road (2000m) and Duggal Bitta $(2,465 \mathrm{~m})$ - Tala Village $(1,700 \mathrm{~m})$. Relative abundance of each species was estimated by adding up the total counts of each individual species for the entire sampling period for all the 10 transects combined. The species counts were then categorized into four groups: rare, uncommon, fairly common and very common, in increasing order of abundance, based on the quartile divisions, from first quartile $(\mathrm{Ql})$ to fourth Quartile $\left(\mathrm{Q}^{4}\right)$, respectively.

Identification of butterflies was done using the following literature: Marshall \& de Nicéville (1882), Moore (1890-1905), Swinhoe (1905-1913), Evans (1932), Talbot (1939, 1947),

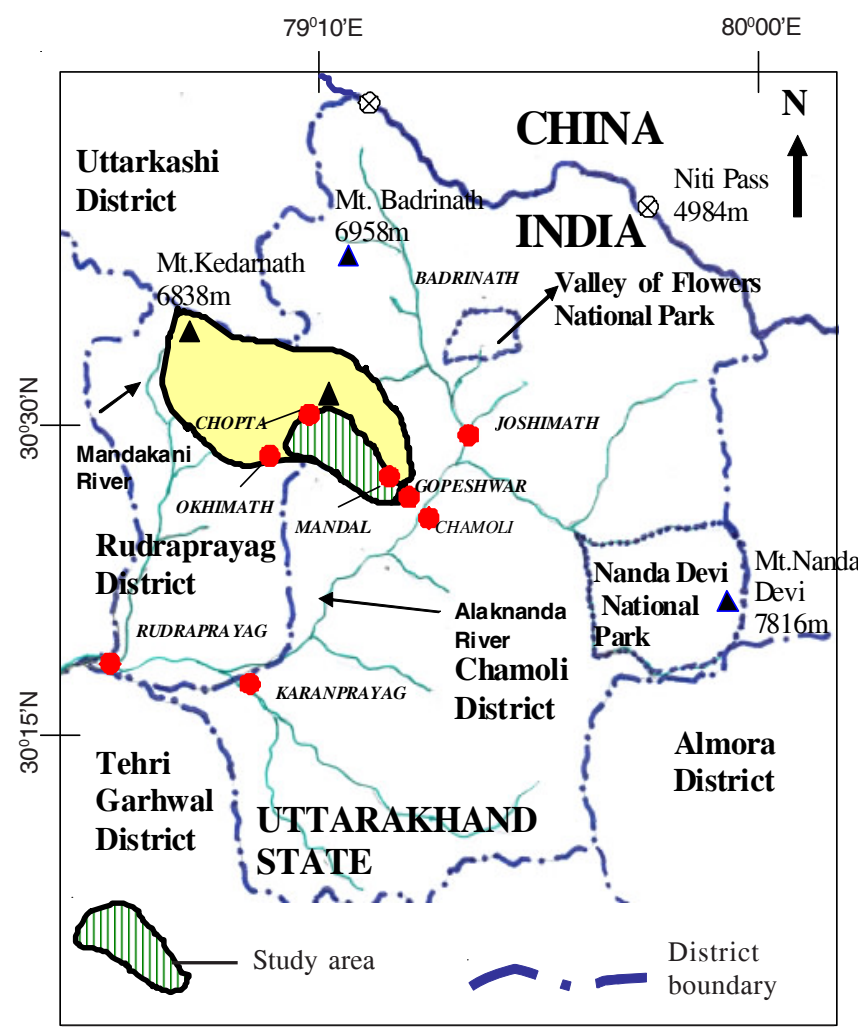

Figure 1. Map of Chamoli and Rudraprayag districts in Garhwal, Uttarakhand state, depicting the location of the study area in Kedarnath Musk Deer Reserve and sites as mentioned in the text.
Wynter-Blyth (1957), D' Abrera (1982, 1985 and 1986), Smith (1989), Haribal (1992) Kunte (2000) and Kehimkar (2008) and also by comparison with identified specimens at the 'National Insect Reference Collection', Entomology Division, Forest Research Institute, Dehradun, India. The classification followed here is based on Ackery (1984).

\section{Results and Discussion}

A total of 3617 individuals of 147 species of butterflies were recorded during eleven sampling surveys carried out in KMDR (Table 1). Butterflies were abundant from late April to September. The individual species count for the entire sampling period for all the transects combined, varied from 1 to 522 individuals. The four groups were then differentiated as 'rare' or Q1 = 1; 'uncommon' or Q2 = 2-4; 'fairly common' or Q3 = 514; 'very common' or $Q_{4}=15-522$, respectively (median $=3$ ).

Findings included one important range extension into the western Himalayas, the Brown Gorgon, Meandrusa lachinus (Fruhstorfer) syn. M. gyas gyas (Westwood), from north-east India (Singh 2006). Besides, the Bi-spot Royal Ancema ctesia ctesia (Hewitson), and the Powdery Green Sapphire Heliophorous tamu tamu (Kollar) were also recorded here west of Kumaon, the previous western limit of the distribution of these species in the Himalaya (Wynter-Blyth 1957; Smith 1989; Varshney 1997). Twelve species (marked with an asterisk in the appendix) are listed in Schedules I, II \& IV of the Indian Wild life (Protection) Act (1972) as amended up to 2006 (Anon 2006). Except for the Lofty Bath White Pontia callidice; Common Silverstripe Fabriciana kamala; Azure Sapphire Heliophorus androcles moorei and Golden Sapphire H.brahma, the remainder of the 35 species recorded from Nanda Devi National Park (Baindur 1993; Uniyal 2004), were recorded in the present study.

The number of species of swallowtails (Papilionidae) in an areas is an indicator of the over-all species richness of butterflies any where in the Indian Sub-continent (Singh \& Pandey 2004). By taking the 'Papilionidae' species proportion as $7.4 \%$ of the total number of species occurring in an area in the Western Himalayas (Singh \& Pandey 2004) the number of species in the study area, where 13 species of 'Papilionidae' were sampled, was estimated to be ca. 176 species as per this methodology. The present figure of 147 species recorded in the study area in KMDR is ca. $83 \%$ of the total number of species estimated in the area. Families, especially, 'Lycaenidae' and 'Hesperiiidae' were under represented, judging from their species proportions of the five major families in the total number of species found in the Western Himalayas (Singh \& Pandey 2004). One reason for recording less number of species than estimated could be that only 10-15 percent of the land area in KMDR was sampled due to inaccessible terrain and low lying areas (below 1,300m) under agriculture and high altitude alpine habitats (above 3,800m) were left out. Although sampling was done during every month from March-mid December, however sampling intensity was low as the total sampling period was only 43 days within the two year study period, which could be another reason for missing a number of species. 
Table 1. Systematic list of butterflies (Rhopalocera) recorded from Kedarnath Musk Deer Reserve, Chamoli and Rudraprayag districts, Uttarakhand, India (May 2006 - September 2008)

\begin{tabular}{|c|c|c|c|c|c|}
\hline & Species & Common Name & Month & Altitudinal range & Relative abundance \\
\hline & $\begin{array}{l}\text { A. Super family: Papilionoidea } \\
\text { I Family: Papilionidae (Swallowtails) } \\
\text { a. Sub-family: Parnassinae } \\
\text { Parnassius hardwickei Gray }\end{array}$ & Common Blue Apollo & June & $3,600 \mathrm{~m}$ & Rare \\
\hline 1 & b. Sub-family: Papilioninae & & & & \\
\hline 2 & Troides aeacus aeacus Felder \& Felder & Golden Birdwing & April-July & $1500-2000 m$ & Fairly common \\
\hline 3 & Atrophaneura polyeuctes letincius Fruhstorfer & Common Windmill & April-May;Sept & $1500 m-1800 m$ & Uncommon \\
\hline 4 & Atrophaneura dasarada ravana Moore & Great Windmill & April-May & $1500 \mathrm{~m}$ & Uncommon \\
\hline 5 & Atrophaneura latreillei latreillei Donovan & Rose Windmill & June-July & $1500-2000 m$ & Very common \\
\hline 6 & Atrophaneura aidoneus Doubleday & Lesser Batwing & April-May; Sept. & $1500-2200 m$ & Uncommon \\
\hline 7 & Papilio protenor protenor Cramer & Spangle & April-May;Sept. & $1500-1800 m$ & Uncommon \\
\hline 8 & Papilio bianor polyctor Boisduval & Common Peacock & April-Sept. & $1500 \mathrm{~m}$ & Fairly common \\
\hline 9 & Papilio polytes romulus Cramer & Common Mormon & Sept.-Oct. & $1500 m$ & Uncommon \\
\hline 10 & Papilio machaon Linnaeus & Common Yellow Swallowtail & Sept.-Oct. & $1500 \mathrm{~m}$ & Uncommon \\
\hline 11 & Pazala eurous cashmirensis Rothschild & Sixbar Swordtail & April-May & $2000-2300 m$ & Fairly common \\
\hline 12 & Graphium cloanthus cloanthus Westwood & Glassy Blue Bottle & April-May; Sept-Oct. & $1500 \mathrm{~m}$ & Fairly common \\
\hline \multirow[t]{2}{*}{13} & Meandrusa lachinus Fruhstorfer* & Brown Gorgon & April-Oct. & $1600-2300 m$ & Fairly common \\
\hline & $\begin{array}{l}\text { II Family: Pieridae (White and Yellows) } \\
\text { a. Sub-family: Pierinae }\end{array}$ & & & & \\
\hline 14 & Pieris daplidice moorei Röber & Bath White & April-Sept. & $1500-1800 m$ & Uncommon \\
\hline 15 & Pieris montana ajaka Moore & Mountain Green-veined White & June-July & $1500-2000 m$ & Very common \\
\hline 16 & Pieris canidia indica Evans & Indian Cabbage White & June-Oct. & $1500-2000 m$ & Very common \\
\hline 17 & Pieris brassicae Linnaeus & Large Cabbage White & April, Oct. & $1500-1700 m$ & Very common \\
\hline 18 & Aporia leucodice soracta Moore & Himalayan Black Vein & June-July & $1500-1800 m$ & Fairly common \\
\hline 19 & Aporia agathon caphusa Moore & Great Black Vein & May-July & $1500 \mathrm{~m}$ & Very common \\
\hline \multirow[t]{2}{*}{20} & Delias belladonna horsfieldii Gray & Hill Jezebel & April-Oct. & $1500-1700 m$ & Fairly common \\
\hline & b. Sub-family: Coliadinae & & & & \\
\hline 21 & Catopsilia pomona Fabricius & Common Emigrant & April; Sept.-Oct. & $1500 \mathrm{~m}$ & Uncommon \\
\hline 22 & Catopsilia pyranthe Linnaeus & Mottled Emigrant & Nov. & $1500 m$ & Uncommon \\
\hline 23 & Gonepteryx rhamni nepalensis Doubleday & Common Brimstone & April-Nov. & $1500-2000 m$ & Very common \\
\hline 24 & Eurema blanda silhetana Wallace & Three Spot Grass Yellow & July, Sept.-Oct. & $1500-1800 m$ & Very common \\
\hline 25 & Eurema brigitta rubella Wallace & Small Grass Yellow & Sept.-Oct. & $1500 \mathrm{~m}$ & Fairly common \\
\hline 26 & Colias fieldii Menétriés & Dark Clouded Yellow & April-Nov. & $1500-3400 m$ & Very common \\
\hline \multirow[t]{2}{*}{27} & Colias erate Esper & Pale Clouded Yellow & March & $1800-2200 m$ & Uncommon \\
\hline & $\begin{array}{l}\text { III Family: Lycaenidae } \\
\text { a. Sub-family: Curetinae }\end{array}$ & & & & \\
\hline \multirow[t]{2}{*}{28} & Curetis bulis Westwood & Bright Sunbeam & June-July;Sept & $1700 m$ & Uncommon \\
\hline & b. Sub-family: Theclinae & & & & \\
\hline 29 & Chrysozephyrus syla Kollar & Silver Hairstreak & Sept. & $2500 \mathrm{~m}$ & Rare \\
\hline 30 & Chrysozephyrus birupa Moore & Fawn Hairstreak & June-July;Sept. & $2000 m$ & Uncommon \\
\hline 31 & Euaspa ziha Hewitson & White Spotted Hairstreak & May & $2100 \mathrm{~m}$ & Rare \\
\hline 32 & Chaetoprocta odata Hewitson & Walnut Blue & May & $1500 \mathrm{~m}$ & Uncommon \\
\hline 33 & Arhopala rama rama Kollar & Dark Himalayan Oakblue & June, Nov. & $1500-2000 m$ & Uncommon \\
\hline 34 & Panchala paraganesa paraganesa de Nicéville & Dusky Bushblue & June-July & $2000 m$ & Uncommon \\
\hline 35 & Spindasis nipalicus nipalicus Moore & Silver-grey Silverline & May & $1500 \mathrm{~m}$ & Uncommon \\
\hline 36 & Tajuria diaeus Hewitson* & Straightline Royal & Nov. & $1500-2000 m$ & Uncommon \\
\hline 37 & Ancema ctesia ctesia Hewitson & Bi-spot Royal & July-Oct. & $1500-1600 m$ & Uncommon \\
\hline 38 & Pratapa iceta icetas Hewitson* & Dark Blue Royal & May & $1800 \mathrm{~m}$ & Rare \\
\hline 39 & Chliaria kina kina Hewitson* & Blue Tit & Oct. & $1650 \mathrm{~m}$ & Rare \\
\hline 40 & Deudorix epijarbas ancus Fruhstorfer & Cornelian & Nov. & $1700 \mathrm{~m}$ & Rare \\
\hline \multirow[t]{2}{*}{41} & Rapala selira Moore & Himalayan Red Flash & April-May & $1800 \mathrm{~m}$ & Very common \\
\hline & c. Sub-family: Lycaeninae & & & & \\
\hline 42 & Lycaena pavana Kollar & White-bordered Copper & April-July; Nov. & $1500-2800 m$ & Fairly common \\
\hline 43 & Lycaena phlaeas indicus Evans & Common Copper & May-June & $3000 \mathrm{~m}$ & Fairly common \\
\hline 44 & Heliophorus tamu tamu Kollar & Powdery Green Sapphire & April-Oct. & $1500-2700 m$ & Very common \\
\hline \multirow[t]{2}{*}{45} & Heliophorus sena Kollar & Sorrel Sapphire & Sept.- Nov. & $1500-1700 m$ & Very common \\
\hline & d. Sub-family: Polyommatinae & & & & \\
\hline 46 & Everes diporides Chapman* & Chapman's Cupid & April;Sept. & $1500-2000 m$ & Uncommon \\
\hline 47 & Acytolepis puspa gisca Fruhstorfer & Common Hedge Blue & June-July; Oct.-Nov. & $1500-2400 m$ & Very common \\
\hline 48 & Celatoxia marginata de Nicéville & Margined Hedge Blue & June & $2000 \mathrm{~m}$ & Fairly common \\
\hline 49 & Udara dilecta Moore & Pale Hedge Blue & July & $1800-2400 m$ & Very common \\
\hline 50 & Udara albocaerulea Moore & Albocerulean & May & $1600 \mathrm{~m}$ & Rare \\
\hline 51 & Celastrina gigas Hemming & Silvery Hedge Blue & April-June; Sept-Oct. & $1500-2400 m$ & Very common \\
\hline 52 & Celastrina huegeli Moore & Large Hedge Blue & Apr.-Oct. & $1500-2200 m$ & Very common \\
\hline 53 & Celastrina argiolus Linnaeus & Hill Hedge Blue & May & $1600 \mathrm{~m}$ & Fairly common \\
\hline 54 & Zizeeria karsandra Moore & Dark Grass Blue & June-July; Sept.-Oct. & $1500-1700 \mathrm{~m}$ & Very common \\
\hline 55 & Pseudozizeeria maha maha Kollar & Pale Grass Blue & Sept-Nov & $1500-2000 m$ & Very common \\
\hline 56 & Lampides boeticus Linnaeus* & Pea Blue & April, July, Nov. & $1500-2000 m$ & Fairly common \\
\hline 57 & Aricia agestis Denis \& Schiffermueller & Orange-bordered Argus & Nov. & $2350 \mathrm{~m}$ & Rare \\
\hline
\end{tabular}




\begin{tabular}{|c|c|c|c|c|c|}
\hline & Species & Common Name & Month & Altitudinal range & Relative abundance \\
\hline & e. Sub-family: Riodininae & & & & \\
\hline 58 & Dodona eugenes eugenes Bates & Tailed Punch & April-May; Oct. & $1800-2000 m$ & Uncommon \\
\hline 59 & Dodona dipoea nostia Fruhstorfer* & Lesser Punch & May-June;Sept & $1400-2,000 m$ & Fairly common \\
\hline 60 & Dodona durga Kollar & Common Punch & May-June; Sept.-Oct. & $1500-1800 m$ & Fairly common \\
\hline 61 & Zemeros flegyas indicus Fruhstorfer & Punchinello & April & $1600-1800 m$ & Rare \\
\hline \multirow[t]{2}{*}{62} & Abisara fylla Doubleday & Dark Judy & May; Sept.-Oct. & $1500-1800 m$ & Fairly common \\
\hline & $\begin{array}{l}\text { IV Family: Nymphalidae } \\
\text { a. Sub family: Satyrinae }\end{array}$ & & & & \\
\hline 63 & Mycalesis nicotia Westwood & Bright-eye Bushbrown & June-July & $1750 \mathrm{~m}$ & Rare \\
\hline 64 & Mycalesis perseus blasius Fabricius & Common Bushbrown & Sept. & $1400-1700 m$ & Fairly common \\
\hline 65 & Mycalesis francisca sanatana Moore & Lilacine Bushbrown & April-May & $2000-2200 m$ & Uncommon \\
\hline 66 & Lethe sidonis vaivarta Doherty & Common Woodbrown & July, Sept. & $1500-1700 m$ & Very common \\
\hline 67 & Lethe maitrya de Nicéville & Barred Woodbrown & June-July & $2000-2500 m$ & Fairly common \\
\hline 68 & Lethe siderea Marshall & Scarce Woodbrown & June-July & $1700-2400 m$ & Uncommon \\
\hline 69 & Lethe insana insana Kollar & Common Forester & May;Sept & $2000-2200 \mathrm{~mm}$ & Uncommon \\
\hline 70 & Lethe verma verma Kollar & Straight-banded Treebrown & June-Sept. & $1500-1800 m$ & Very common \\
\hline 71 & Lethe baladeva aisa Fruhstorfer & Treble Silverstripe & June & 2000m & Rare \\
\hline 72 & Zophoessa goalpara narkanda Fruhstorfer & Large Goldenfork & Sept. & $2200 m$ & Rare \\
\hline 73 & Neope yama yama Moore & Dusky Labyrinth & Sept. & $2000 m$ & Rare \\
\hline 74 & Neope pulaha pulaha Moore & Veined Labyrinth & April -July & $1500-1800 m$ & Uncommon \\
\hline 75 & Melanitis phedima galkissa Fruhstorfer & Dark Evening Brown & Oct. & $1600 \mathrm{~m}$ & Rare \\
\hline 76 & Dallacha hyagriva hyagriva Moore & Brown Argus & Sept. & $1700 \mathrm{~m}$ & Rare \\
\hline 77 & Callerebia annada caeca Watkins & Ringed Argus & May-July;Sept & $1600-2500 m$ & Very common \\
\hline 78 & Callerebia hybrida Butler? & Hybrid Argus & June-July & $1500-2300 m$ & Very common \\
\hline 79 & Callerebia scanda scanda Kollar & Pallid Argus & Sept.-Oct. & $1800-2000 m$ & Uncommon \\
\hline 80 & Callerebia nirmala nirmala Moore & Common Argus & July & $1500 \mathrm{~m}$ & Very common \\
\hline 81 & Ypthima nareda nareda Kollar & Large Three Ring & May;Sept & $1700-2000 m$ & Uncommon \\
\hline 82 & Ypthima sakra nikaea Moore & Himalayan Five Ring & April-Oct. & $1500-2300 m$ & Very common \\
\hline 83 & Ypthima baldus baldus Fabricius & Common Five Ring & June & $1500-1700 m$ & Uncommon \\
\hline 84 & Ypthima indecora Moore & Western Five Ring & March-May;Sept & $1500-2200 m$ & Fairly common \\
\hline 85 & Orinoma damaris Gray & Tiger Brown & July-Nov. & $1600-2000 m$ & Fairly common \\
\hline 86 & Raphicera mooreiButler & Small Tawny Wall & July & $2300 \mathrm{~m}$ & Uncommon \\
\hline 87 & Pararge schakra Kollar & Common Wall & June-Oct. & $1700-2500 m$ & Fairly common \\
\hline 88 & Aulocera swaha swaha Kollar & Common Satyr & April-Oct. & $1800-2000 m$ & Very common \\
\hline 89 & Aulocera loha Doherty & Great Satyr & July-Sept. & $2000 \mathrm{~m}$ & Fairly common \\
\hline \multirow[t]{2}{*}{90} & Aulocera brahminus dokwana Evans & Narrow Banded Satyr & July- Sept. & $3,500 m$ & Rare \\
\hline & b. Sub-family: Charaxinae & & & & \\
\hline \multirow[t]{2}{*}{91} & Polyura dolon dolon Westwood & Stately Nawab & May & $2700 \mathrm{~m}$ & Rare \\
\hline & c. Sub-family: Apaturinae & & & & \\
\hline 92 & Dilipa morgiana Westwood * & Golden Emperor & March & $1600 \mathrm{~m}$ & Rare \\
\hline 93 & Sephisa dichroa Kollar & Western Courtier & May-Oct. & $1500-2000 m$ & Fairly common \\
\hline 94 & Apatura ambica ambica Kollar & Indian Purple Emperor & Sept. & $1700 \mathrm{~m}$ & Rare \\
\hline 95 & Dichorragia nesimachus Boisduval & Constable & May & $1500-2400 m$ & Rare \\
\hline \multirow[t]{2}{*}{96} & Stibochiona nicea nicea Gray & Popinjay & April-May; Sept.-Oct. & $1600-1700 m$ & Uncommon \\
\hline & d. Sub-family: Nymphalinae & & & & \\
\hline 97 & Auzakia danava Moore & Commodore & April-May & $1600-2000 m$ & Uncommon \\
\hline 98 & Athyma asura asura Moore & Studded Sergeant & Sept.-Oct. & $2000 \mathrm{~m}$ & Rare \\
\hline 99 & Athyma selenophora Moore & Staff Sergeant & Nov. & $1500 \mathrm{~m}$ & Rare \\
\hline 100 & Athyma opalina opalina Kollar & Himalayan Sergeant & April-Nov. & $1500-2400 m$ & Very common \\
\hline 101 & Neptis ananta ananta Moore & Yellow Sailer & April-July & $1500-1800 m$ & Fairly common \\
\hline 102 & Neptis soma butleri Eliot & & April-May; Sept.-Oct. & $1500-2000 m$ & Very common \\
\hline 103 & Neptis mahendra Moore & Himalayan Sailer & April-Oct. & $1500-2000 m$ & Very common \\
\hline 104 & Neptis sappho astola Moore & Pallas' Sailer & April & $1500-2000 m$ & Common \\
\hline 105 & Neptis sankara sankara Kollar & Broad-banded Sailer & May;Sept & $1400-1800 \mathrm{~mm}$ & Uncommon \\
\hline 106 & Cyrestis thyodamas ganescha Kollar & Common Map & May-Oct. & $1500-1700 m$ & Fairly common \\
\hline 107 & Hypolimnas misippus Linnaeus & Danaid Eggfly & Sept. & $1700 \mathrm{~m}$ & Uncommon \\
\hline 108 & Kallima inachus huegeli Kollar & Orange Oakleaf & April-Sept. & $1500-1700 m$ & Uncommon \\
\hline 109 & Psuedergolis wedah Kollar & Tabby & May-June; Sept.-Oct. & $1500-2000 m$ & Fairly common \\
\hline 110 & Phalantha phalantha Drury & Common Leopard & September & $1700 m$ & Rare \\
\hline 111 & Precis iphita iphita Cramer & Chocolate Soldier & April-Oct. & $1500-2000 m$ & Very common \\
\hline 112 & Cynthia cardui Linnaeus & Painted Lady & April-June; Nov. & $1500-3700 m$ & Very common \\
\hline 113 & Kaniska canace himalaya Evans & Blue Admiral & June-Oct. & $1500-3000 m$ & Uncommon \\
\hline 114 & Vanessa indica indica Herbst & Indian Red Admiral & April-June; Sept.-Nov. & $.1500-1800 m$ & Very common \\
\hline 115 & Aglais cashmirensis aesis Fruhstorfer & Indian Tortoiseshell & April-May; Sept.-Oct. & $1500-3700 m$ & Very common \\
\hline 116 & Nymphalis xanthomelas fervescens Stichel & Large Tortoiseshell & April-May & $3600 \mathrm{~m}$ & Uncommon \\
\hline 117 & Polygonia egea agnicula Moore & Eastern Comma & August-Oct. & $2700-3500 m$ & Uncommon \\
\hline 118 & Symbrenthia lilaea khasiana Moore & Common Jester & May; Sept.-Nov. & $1500-1800 m$ & Fairly common \\
\hline 119 & Symbrenthia hypselis cotanda Moore & Spotted Jester & April-Oct. & $1500-2000 m$ & Fairly common \\
\hline 120 & Symbrenthia brabira Moore & Himalayan Jester & April-Oct. & $1500-1800 m$ & Uncommon \\
\hline 121 & Argyreus hyperbius hyperbius Linnaeus & Indian Fritillary & April & $1700 \mathrm{~m}$ & Fairly common \\
\hline 122 & Childrena childreni Gray & Large Silverstripe & Sept.-Oct. & $1500-1800 m$ & Rare \\
\hline 123 & Issoria issaea Moore & Queen of Spain Fritillary & April-July & $1700-3500 m$ & Fairly common \\
\hline 124 & Euthalia patala patala Kollar & Grand Duchess & June-July & $1600-2000 m$ & Fairly common \\
\hline
\end{tabular}




\begin{tabular}{|c|c|c|c|c|c|}
\hline & Species & Common Name & Month & Altitudinal range & Relative abundance \\
\hline 125 & $\begin{array}{l}\text { e. Sub-family: Acraeinae } \\
\text { Pareba vesta Fabricius }\end{array}$ & Yellow Coster & Aug.-Sept. & $1400-1500 m$ & Uncommon \\
\hline $\begin{array}{l}126 \\
127 \\
128 \\
129\end{array}$ & $\begin{array}{l}\text { f. Sub-family: Danainae } \\
\text { Parantica sita Kollar } \\
\text { Danaus genutia Cramer } \\
\text { Euploea mulciber mulciber Cramer* } \\
\text { Euploea core core Cramer }\end{array}$ & $\begin{array}{l}\text { Chestnut Tiger } \\
\text { Common Tiger } \\
\text { Striped Blue Crow } \\
\text { Common Indian Crow }\end{array}$ & $\begin{array}{l}\text { April-Oct. } \\
\text { May } \\
\text { April-July } \\
\text { Sept.-Oct. }\end{array}$ & $\begin{array}{l}1500-2200 \mathrm{~m} \\
1500 \mathrm{~m} \\
1500 \mathrm{~m} \\
1500 \mathrm{~m}\end{array}$ & $\begin{array}{l}\text { Very common } \\
\text { Rare } \\
\text { Uncommon } \\
\text { Rare }\end{array}$ \\
\hline 130 & $\begin{array}{l}\text { g. Sub-family: Libytheinae } \\
\text { Libythea lepita lepita Moore* }\end{array}$ & Common Beak & April-May & $1500-1800 m$ & Uncommon \\
\hline & $\begin{array}{l}\text { B. Super Family: Hesperioidea } \\
\text { I Family: Hesperiidae } \\
\text { a. Sub-family: Coeliadinae }\end{array}$ & & & & \\
\hline 131 & Bibasis jaina jaina Moore & Orange Awlet & Nov. & $1700 \mathrm{~m}$ & Rare \\
\hline 132 & $\begin{array}{l}\text { Choaspes benjaminii xanthropogon Kollar } \\
\text { b. Sub-family: Pyrginae }\end{array}$ & Indian Awlking & Sept. & $1800-2100 m$ & Uncommon \\
\hline 133 & Celaenorrhinus leucocera leucocera Kollar & Common Spotted Flat & June-July & $2000-2500 m$ & Uncommon \\
\hline 134 & Celaenorrhinus dhanada Moore & Himalayan Yellow Flat & Sept. & $2000 \mathrm{~m}$ & Rare \\
\hline 135 & Celaenorrhinus pero pero de Nicéville & Mussoorie Spotted Flat & June-July & $1500-2500 m$ & Very common \\
\hline 136 & Tagiades menaka Moore & Spotted Snow Flat & May-July & $1500-2000 m$ & Uncommon \\
\hline 137 & $\begin{array}{l}\text { Pseudocoladenia dan fatih Kollar } \\
\text { c. Sub-family: Hesperiinae }\end{array}$ & Fulvous Pied Flat & June; Sept.-Oct. & $1500-2000 m$ & Fairly common \\
\hline 138 & Aeromachus dubius dubius Elwes \& Edwards & Dingy Scrub Hopper & Sept. & $1700-800 \mathrm{~m}$ & Uncommon \\
\hline 139 & Sovia grahami Evans & Graham's Ace & May & $1700 \mathrm{~m}$ & Rare \\
\hline 140 & Pedesta masuriensis masuriensis Moore & Mussoorie Bush Bob & May & $1800 \mathrm{~m}$ & Rare \\
\hline 141 & Notocrypta feisthamelii alysos Moore & Spotted Demon & April-July;Sept & $1500-2500 m$ & Fairly common \\
\hline 142 & Potanthus dara Kollar & Himalayan Grass Dart & May & $1700 \mathrm{~m}$ & Rare \\
\hline 143 & Potanthus pseudomaesa clio Evans & Indian Dart & Sept & $1500-1800 m$ & Common \\
\hline 144 & Parnara guttatus guttatus Brem. & Straight Swift & Sept. & $1500 \mathrm{~m}$ & Rare \\
\hline 145 & Polytremis discreta discreta Elwes \& Edwards* & Himalayan Swift & July & $1500-1800 m$ & Rare \\
\hline 146 & Polytremis eltola Hewitson & Yellow Spot Swift & April-May; Sept.-Oct. & $1500-1700 m$ & Common \\
\hline 147 & Pelopias mathias mathias Fabricius & Small-branded Swift & July & $1700 \mathrm{~m}$ & Uncommon \\
\hline
\end{tabular}

\section{References}

Anonymous (2006). The Wildlife (Protection) Act 1972. Natraj Publishers, Dehradun, 235pp.

Ackery, P.R. (1984). Systematic and Faunistic Studies on Butterflies, pp.2-91. In: VaneWright, R.I. \& P.R. Ackery (eds.). The Biology of Butterflies No: 11. Symposium of the Royal Entomological Society of London, Academic Press.

Baindur, A. (1993). The butterflies of Nanda Devi, pp.35-43. In: Scientific and Ecological Expedition Nanda Devi, 2nd May to 22nd July 1993. (Army Corps of Engineers; W.I.I.; Salim Ali Centre for Ornithology \& Natural History, WWF-India, G.B.Pant Institute of Himalayan Environment \& Development, Botanical Survey of India) Army Headquarters, New Delhi.

Champion, H.G. \& S.K. Seth (1968). Forest Types of India. Government of India Publication, Delhi, 404pp.

D'Abrera, B. (1982). Butterflies of the Oriental Region. Part I. Papilionidae, Pieridae \& Danaidae. Hill House, Victoria, Australia, 244pp.

D'Abrera, B. (1986). Butterflies of The Oriental Region. Part II Nymphalidae, Satyridae \& Amathusiidae. Hill House, Victoria, Australia, 534pp.

D'Abrera, B. (1998). Butterflies of The Oriental Region. Part III. Lycaenidae \& Riodinidae. Hill House, Victoria, Australia, 672pp.

Doherty, W. (1886). A list of butterflies of Kumaon. Journal of Asiatic Society of Bengal 55(2): 103-140.

Evans, W.H. (1932). The Identification of Indian Butterflies - 2nd edition. Bombay Natural History Society, Bombay, 464pp.

Green, J.B.M. (1986). The birds of Kedarnath Sanctuary, Chamoli district, Uttar Pradesh: status and distribution. Journal of the Bombay Natural History Society 83: 603-617.

Hannyngton, F. (1910). The butterflies of Kumaon. Parts I \& II. Journal of the Bombay Natural History Society 20: 130-142; 361-372.

Haribal, M. (1992). The Butterflies of Sikkim Himalaya and their Natural History. Sikkim Nature Conservation Foundation, Sikkim, 217pp.

Kehimkar, I. (2008). The Book of Indian Butterflies. Bombay Natural
History Society. Oxford University Press. Mumbai, pp497.

Kunte, K. (2000). Butterflies of Peninsular India. Universities Press, Hyderabad, 254pp.

Mackinnon, P.W. \& L. De Nicéville (1899). List of butterflies of Mussoorie in the Western Himalayas and neighbouring region. Journal of the Bombay Natural History Society 11: 205-221, 368-389, 585-605.

Marshall, G.F.L. \& L. de Nicéville (1882). Butterflies of India, Burmah and Ceylon. Vol.-1. Central Press, Calcutta, $321 \mathrm{pp}$.

Moore, F. (1890-1992). Lepidoptera Indica Part I. Rhopalocera-Nymphalidae. Lovell, Reeve \& Co.Ltd, London, 317pp + 1-94pls.

Moore, F. (1893-1896). Lepidoptera Indica Part II. Rhopalocera-Nymphalidae. Lovell, Reeve \& Co.Ltd, London, 274pp + 95-190pls.

Moore, F. (1896-1899). Lepidoptera Indica Part III. RhopaloceraNymphalidae. Lovell, Reeve \& Co.Ltd, London, 254pp + 191-286pls.

Moore, F. (1899-1900). Lepidoptera Indica Part IV. RhopaloceraNymphalidae. Lovell, Reeve \& Co.Ltd, London, 260pp + 287-378pls.

Moore, F. (1901-1903). Lepidoptera Indica Parts V. RhopaloceraNymphalidae, Riodininae \& Paplionidae. Lovell, Reeve \& Co.Ltd, London, $248 \mathrm{pp}+379-416 \mathrm{pls}$.

Moore, F. (1903-1905). Lepidoptera Indica Parts VI. Rhopalocera- Paplionidae \& Pieridae. Lovell, Reeve \& Co.Ltd, London, 240pp + 467-550pp.

Myers, S. \& A.P. Singh (2006). Birding hotspot: Kedarnath Musk Deer Reserve, Uttaranchal, India. Birding Asia 5: 60-68.

Ollenbach, O.C. (1930). Butterfly collection grounds at Mussoorie (U.P.). Journal of the Bombay Natural History Society 34: 836-840.

Pande, P.K., J.D.S. Negi \& S.C.Sharma (2001). Plant species diversity and vegetation analysis in a moist temperate Himalayan forest. Indian Journal of Forestry 24: 456-470.

Pollard, E, D.O. Elias, M.J. Skelton \& J.A. Thomas (1975). A method for assessing the abundance of butterflies in Monk's Wood National Nature Reserve in 1973. Entomologist's Gazette 26: 79-88.

Semwal, J.K. \& R.D. Gaur (1981). Alpine flora of Tungnath in the Garhwal Himalaya. Journal of the Bombay Natural History Society 78: 498-512. 


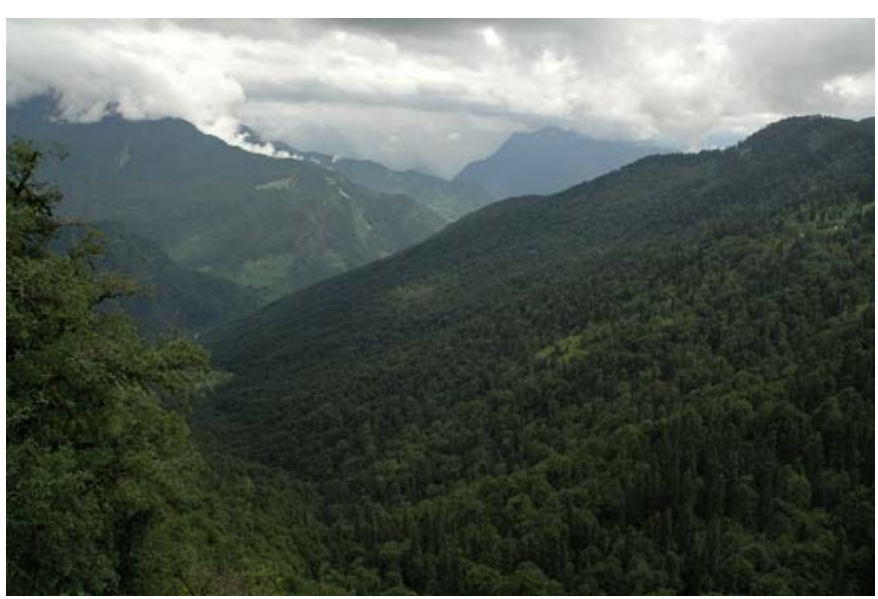

Image 1. Moist temperate forest

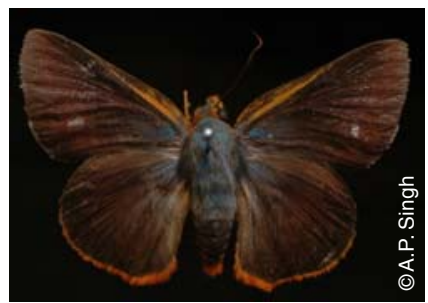

Image 3. Orange Awlet, Bibasis jaina (upper)

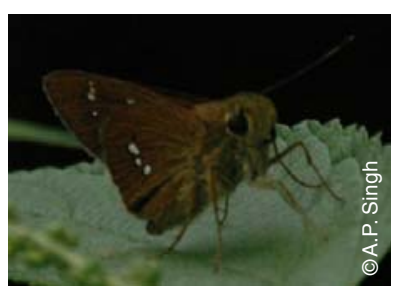

Image 7. Yellow-spot

Swift,Polytremis eltola (under)

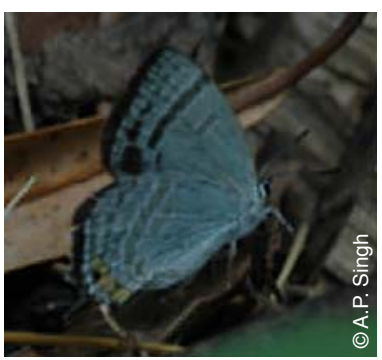

Image 11. White Spotted Hairstreak Euaspa ziha (under)

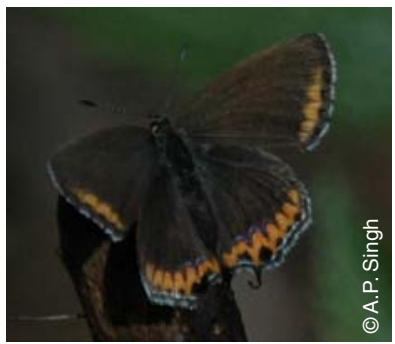

Image 15. Sorrel Sapphire, Heliophorus sena (upper)

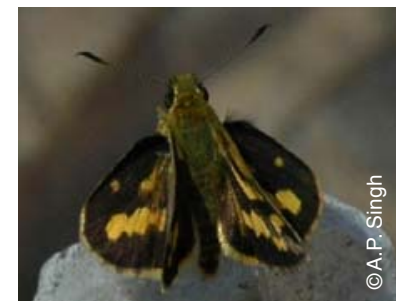

Image 4. Himalayan Dart, Potanthus dara (upper)

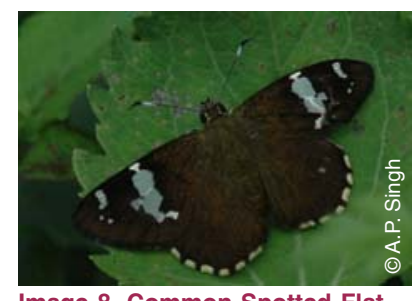

Image 8. Common Spotted Flat, Celaenorrhinus leucocera (upper)

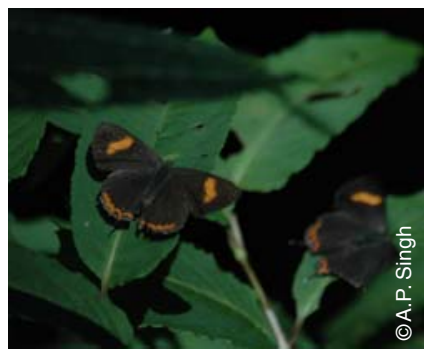

Image 12. Powdery Green Sapphire, Heliophorus tamu - females

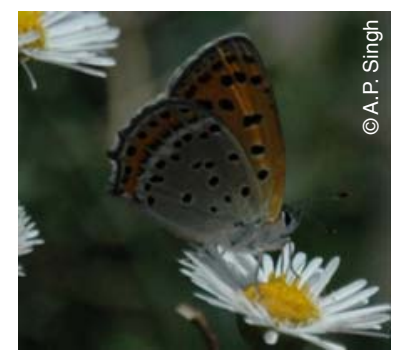

Image 16. White-bordered Copper, Lycaena pavana

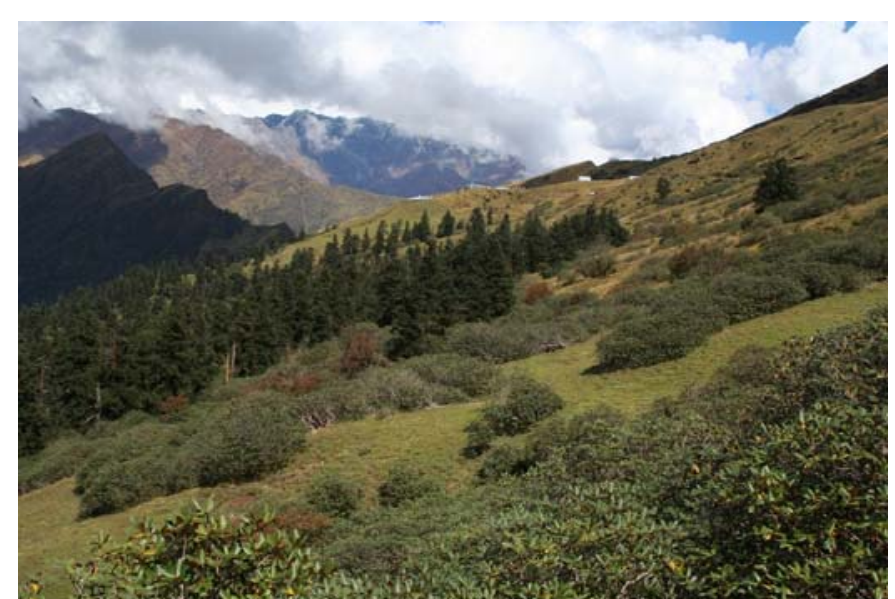

Image 2. Sub-alpine zone with Rhododendron campanulatum shrubberies

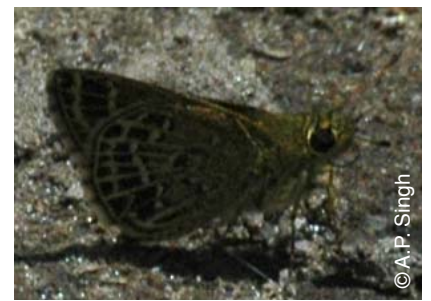

Image 5. Mussoorie Bush Bob, Pedesta masuriensis (under)

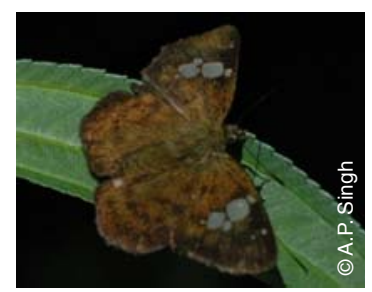

Image 9. Fulvous-pied Flat,

Pseudocoladenia dan fatih (upper)

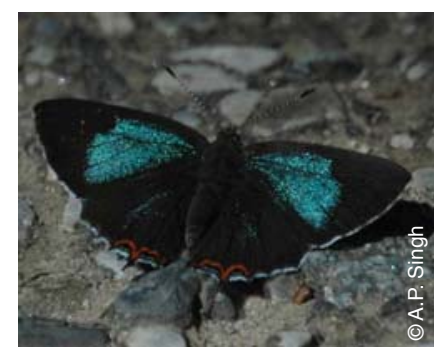

Image 13. Powdery Green

Sapphire, Heliophorus tamu - male

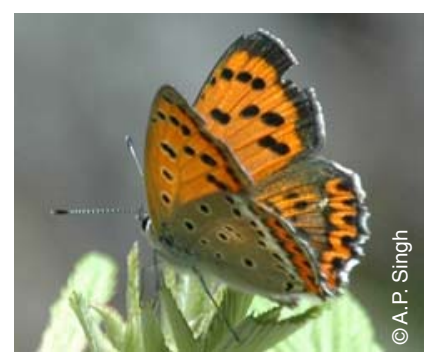

Image 17. White-bordered Copper, Lycaena pavana 2

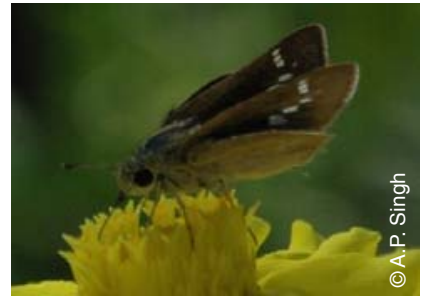

Image 6. Striaight Swift, Parnara guttatus (upper)

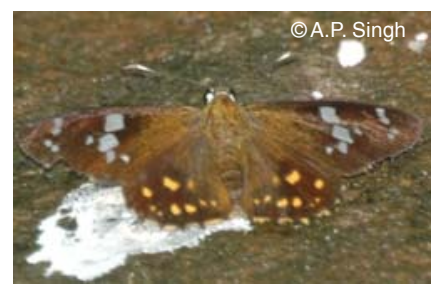

Image 10. Mussoorie Spotted Flat, Celaenorrhinus pero (upper)

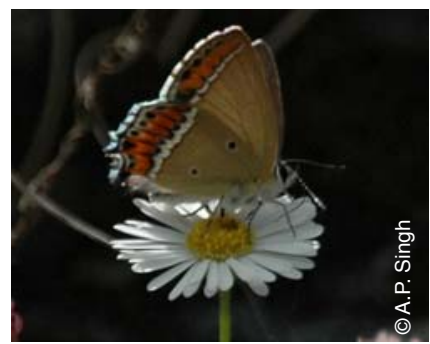

Image 14. Sorrel Sapphire, Heliophorus sena (under)

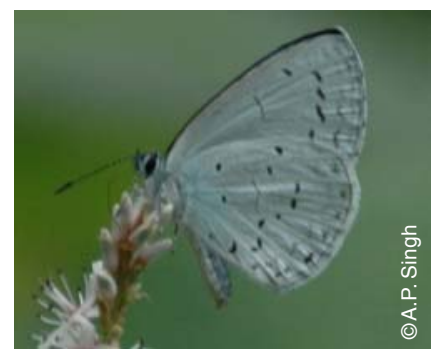

Image 18. Albocerulean, Udara albocaerulea (under) 


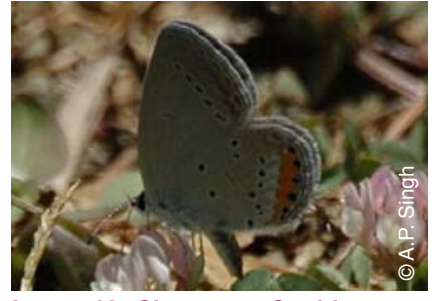

Image 19. Chapmans Cupid, Everes diporides

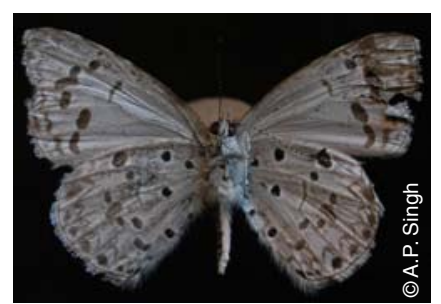

Image 23. Margined Hedge Blue, Celatoxia marginata -male (under)

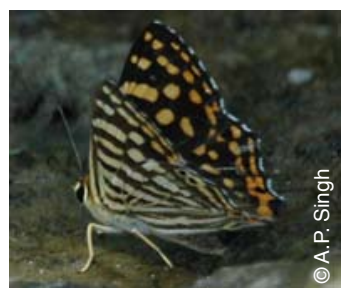

Image 27. Common Punch, Dodona durga

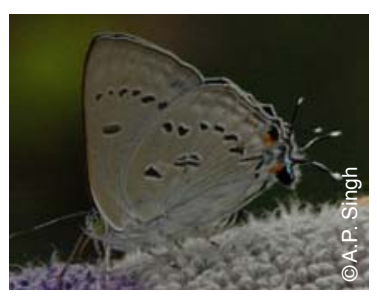

Image 31. Bi-Spot Royal, Ancema ctesia (under)

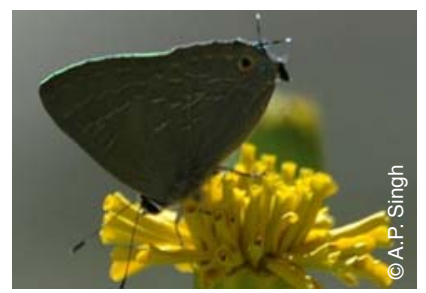

Image 35. Cornelian, Deudorix epijarbas (under)

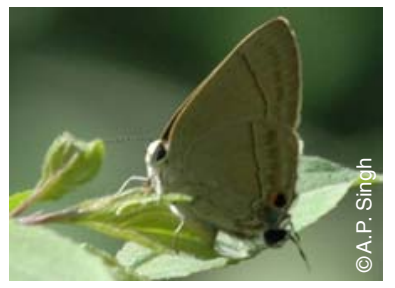

Image 39. Himalayan Red Flash, Rapala selira (under)

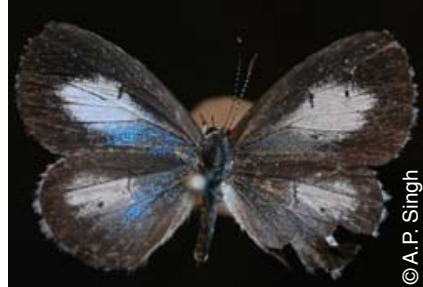

Image 20. Common Hedge Blue, Acytolepis puspa -female (upper)

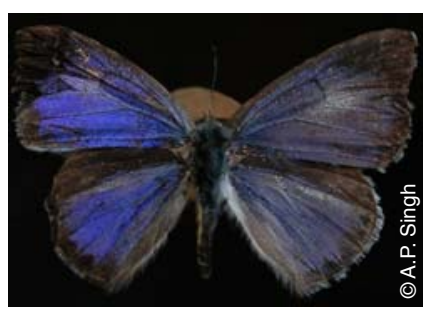

Image 24. Margined Hedge Blue, Celatoxia marginata (upper)

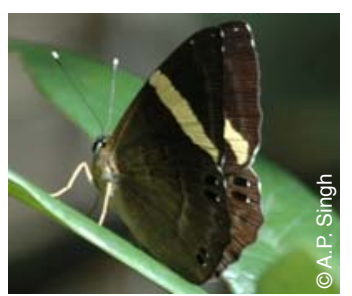

Image 28. Dark Judy, Abisara fylla

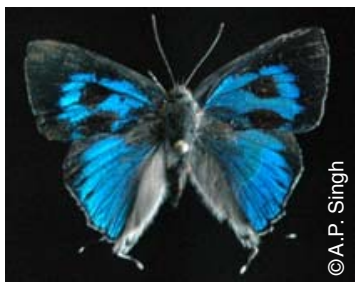

Image 32. Bi-spot Royal, Ancema ctesia (upper)

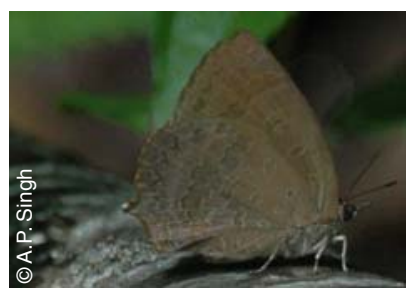

Image 36. Dark Himalayan Oakblue, Arhopala rama

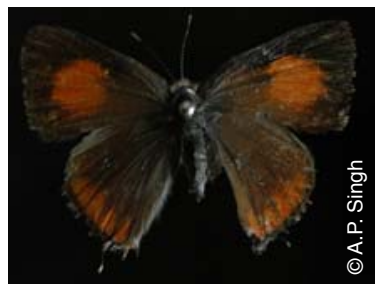

Image 40. Himalayan Red Flash, Rapala selira (upper)

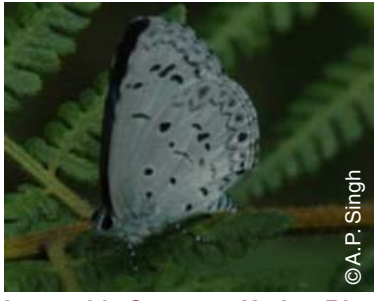

Image 21. Common Hedge Blue, Acytolepis puspa (under)

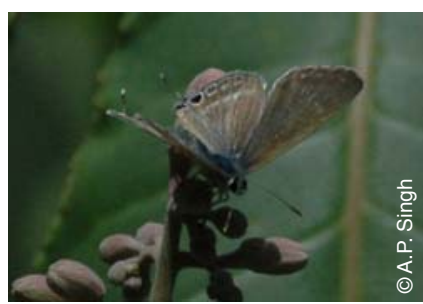

Image 25. Pea Blue, Lampides boeticus (upper)

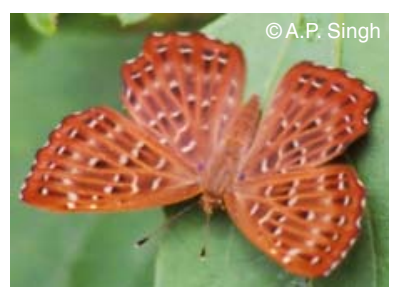

Image 29. Punchinello, Zemeros flegyas (upper)

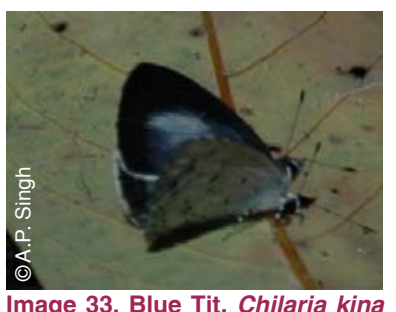

Image 33. Blue Tit, Chilaria kina

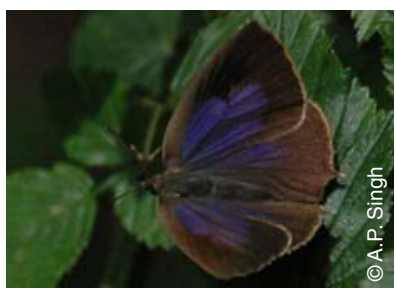

Image 37. Dark Himalayan Oakblue, Arhopala rama (upper)

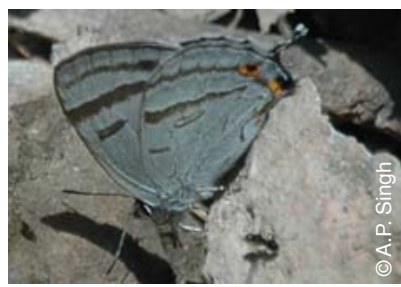

Image 41. Silver Hairstreak, Chrysozephyrus syla male

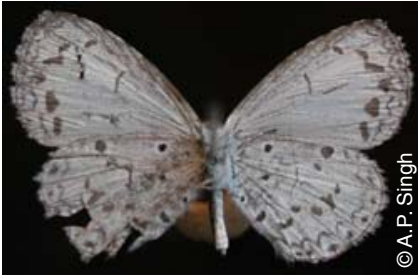

Image 22. Common Hedgeblue, Acytolepis puspa - female (under)

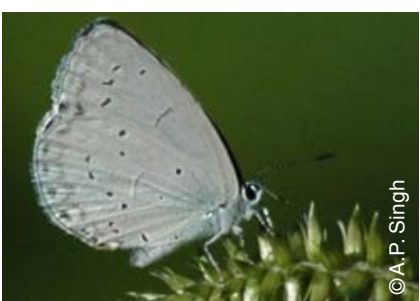

Image 26. Silvery Hedge Blue, Celastrina gigas (under)

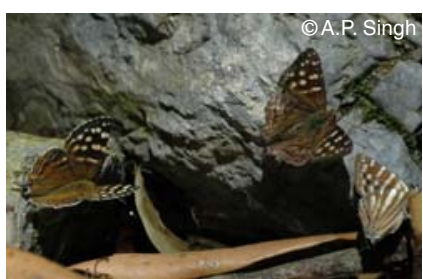

Image 30. Tailed Punch, Dodona eugenes

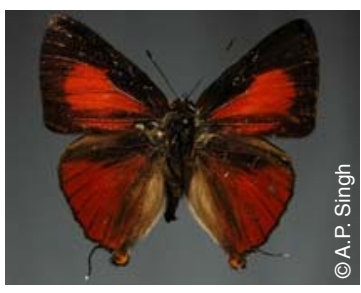

Image 34. Cornelian, Deudorix epijarbas (upper)

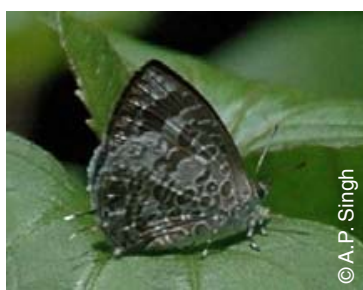

Image 38. Dusky Bush Blue, Panchala paraganesa (under)

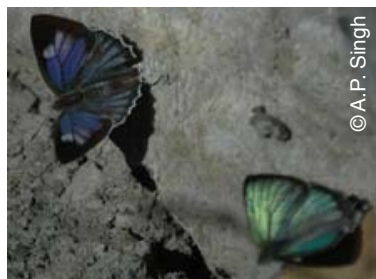

Image 42. Silver Hairstreak, Chrysozephyrus syla M \& F 


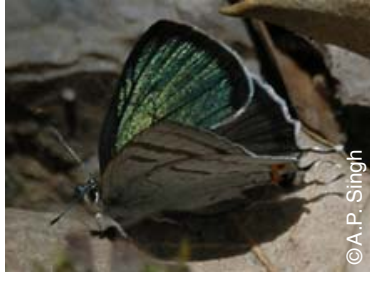

Image 43. Silver Hairstreak Chrysozephyrus syla male - 3

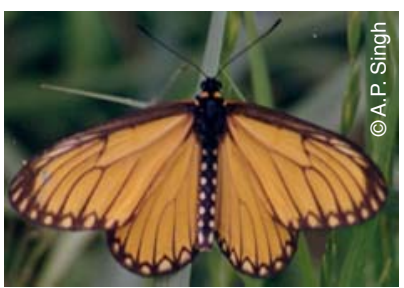

Image 47. Yellow Coster, Pareba vesta (upper)

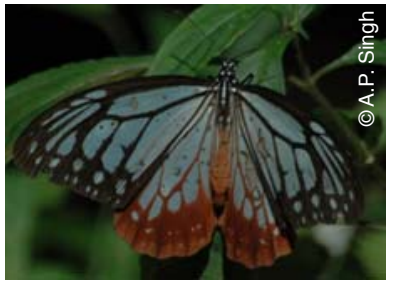

Image 51. Chestnut Tiger,

Parantica sita

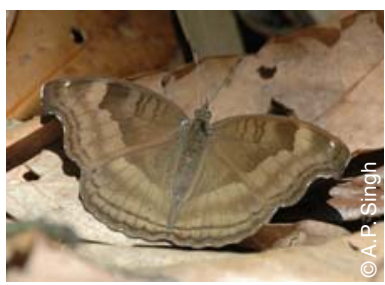

Image 55. Chocolate Soldier, Precis iphita (upper)

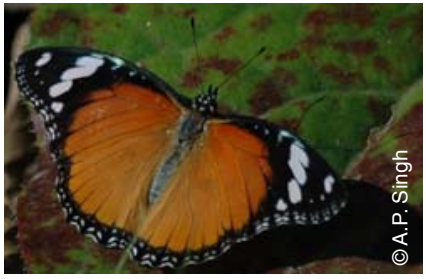

Image 59. Danaid Eggfly, Hypolimnas misippus (form inaria) (upper)

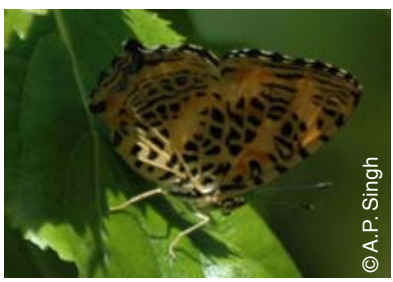

Image 63. Himalayan Jester Symbrenthia brabira

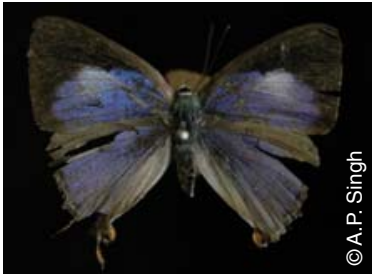

Image 44. Straightline Royal, Tajuria diaeus Female (upper)

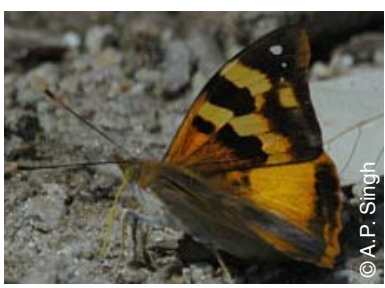

Image 48. Golden Emperor, Dilipa morgiana (upper)

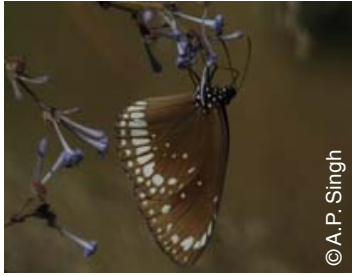

Image 52. Common Indian Crow, Euploea core

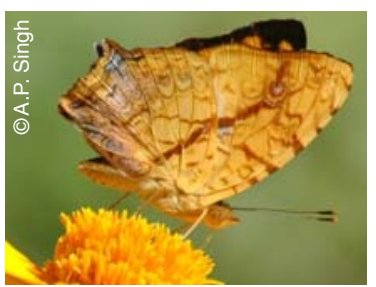

Image 56. Common Jester, Symbrenthia Iilaea (under)

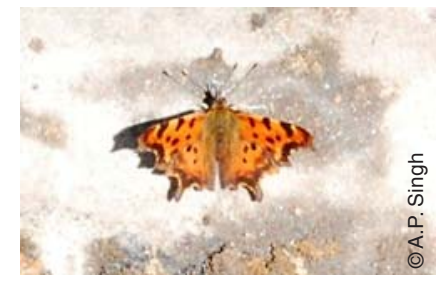
Image 60. Eastern Comma, Polygonia egea agnicula (upper)

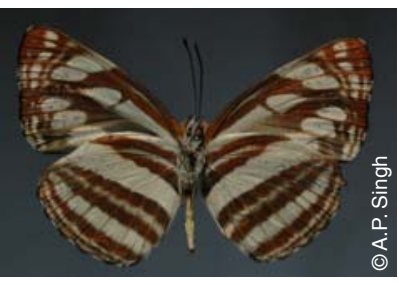

Image 64. Himalayan Sailer, Neptis mahendra (under)

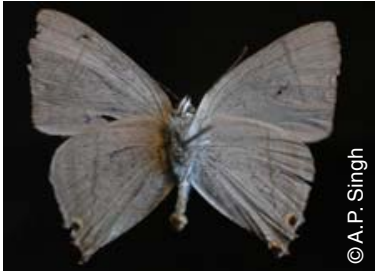

Image 45. Straightline Royal, Tajuria diaeus Male (under)

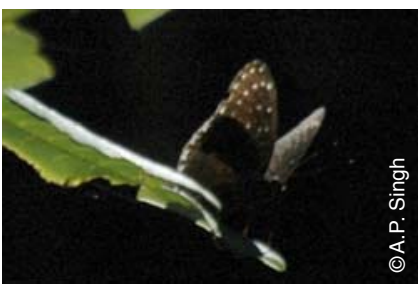

Image 49. Popinjay, Stibochiona nicea (under)

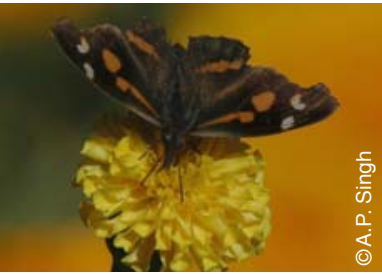

Image 53. Common Beak, Libythea lepita (upper)

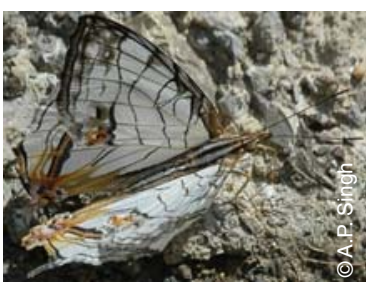

Image 57. Common Map, Cyrestis thyodamas (upper)

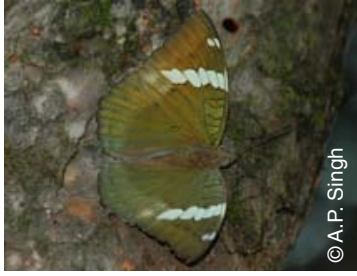

Image 61. Grand Duchess, Euthalia patala (upper)

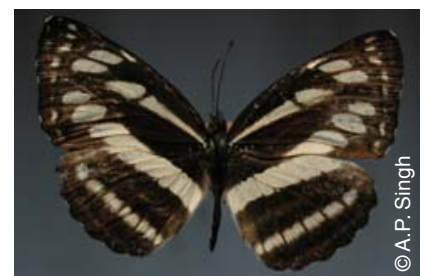

Image 65. Himalayan Sailer, Neptis mahendra (upper)

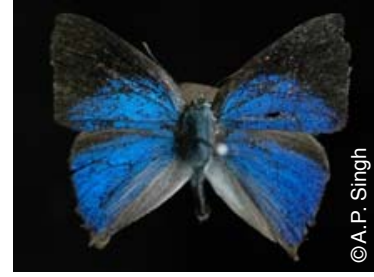

Image 46. Straightline Royal, Tajuria diaeus Male (upper)

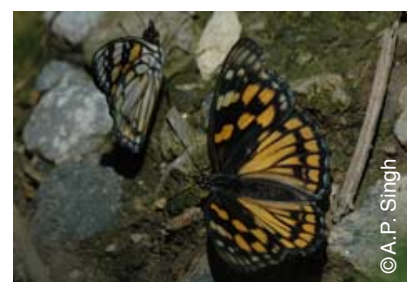

Image 50. Western Courtier, Sephisa dichroa

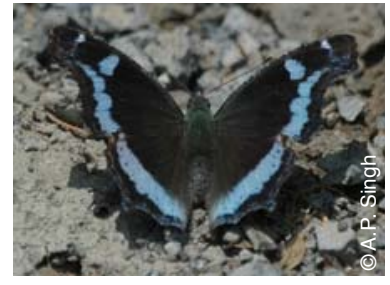

Image 54. Blue Admiral, Kaniska canace (upper)

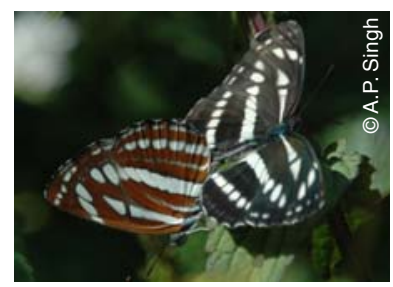

Image 58. Pallas' Sailor, Neptis sappho astola (upper)

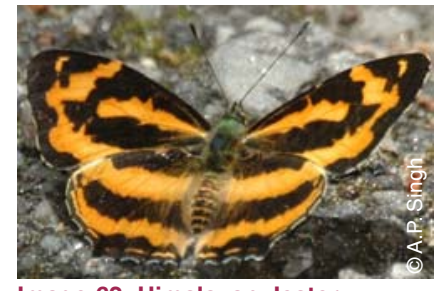

Image 62. Himalayan Jester,

Symbrenthia brabira (upper)

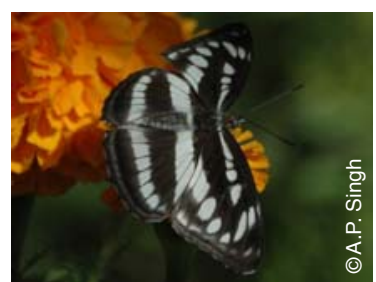

Image 66. Himalayan Sergeant, Athyma opalina (upper) 


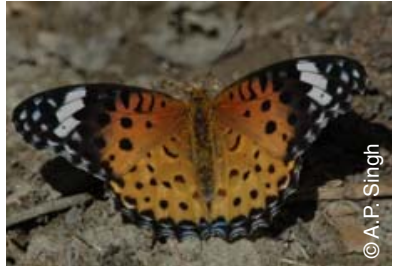

Image 67. Indian Fritillary,

Argyreus hyperbius female (upper)

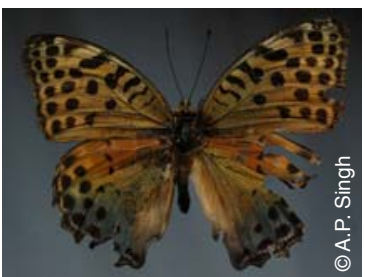

Image 71. Large Silverstripe, Childrena childreni

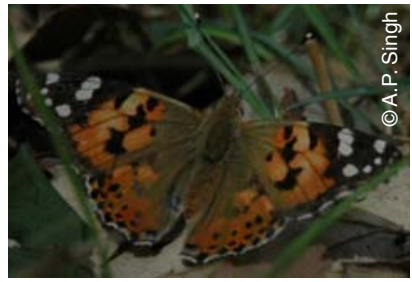

Image 75. Painted Lady, Cynthia cardui (upper)

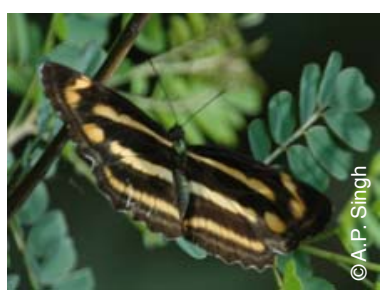

Image 79. Yellow Sailer, Neptis ananta (upper)

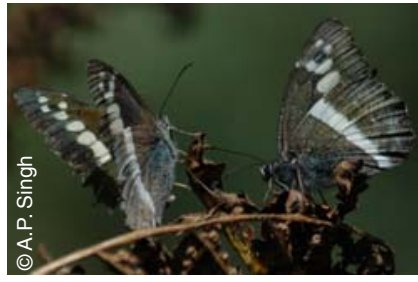

Image 83. Common Satyr,

Aulocera swaha

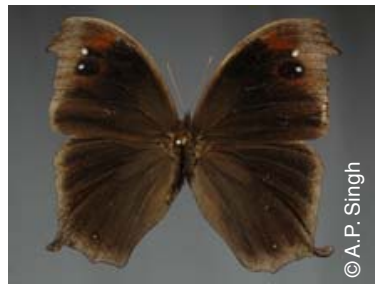

Image 87. Dark Evening Brown, Melanitis phedima (upper)

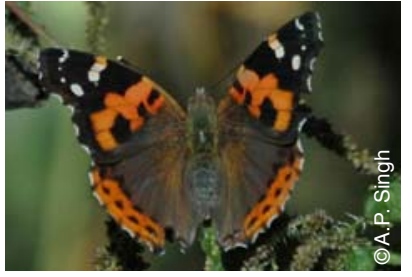

Image 68. Indian Red Admiral,

Vanessa indica (upper)

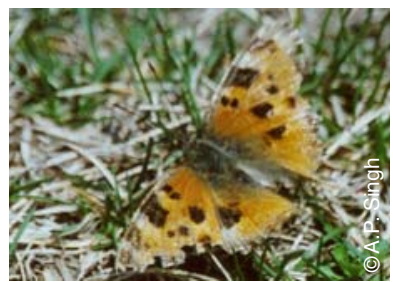

Image 72. Large Tortoiseshell,

Nymphalis xanthomelas (upper)

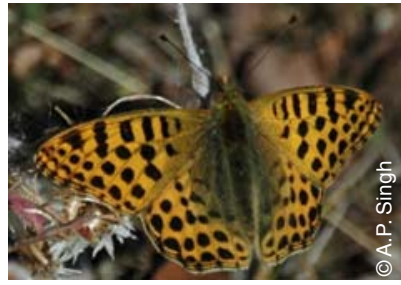

Image 76.Queen of Spain Fritillary, Issoria lathonia (upper)

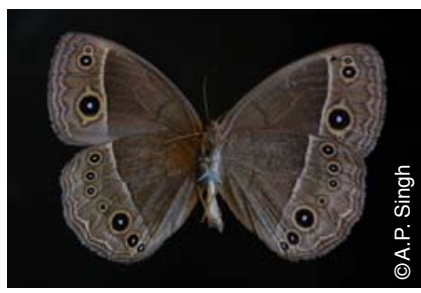

Image 80. Bright-eye Bushbrown, Mycalesis nicotia (under)

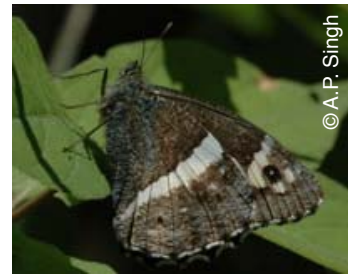

Image 84. Common Satyr

Aulocera swaha (under)

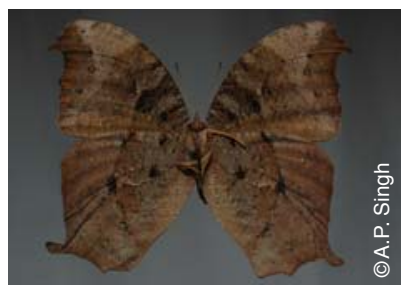

Image 88. Dark Evening Brown, Melanitis phedima (under)

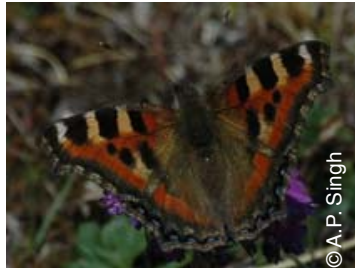

Image 69. Indian Tortoiseshell, Aglais cashmirensis (upper)

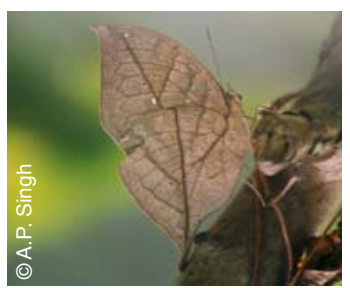

Image 73. Orange Oak Leaf, Kallima inachus (under)

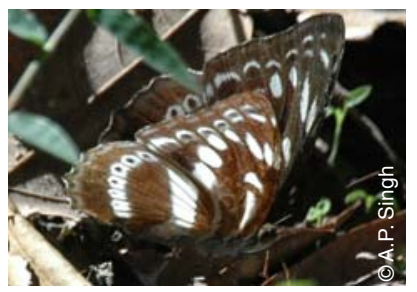

Image 77. Studded Sergeant, Athyma asura (under)

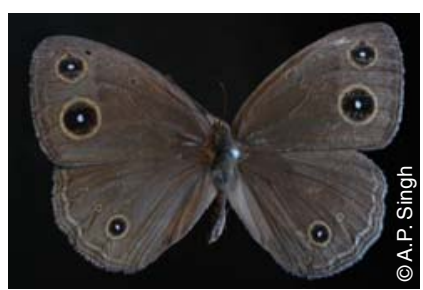

Image 81. Bright-eye Bushbrown, Mycalesis nicotia (upper)

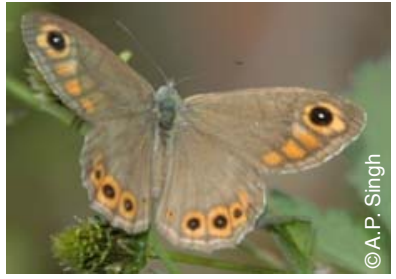

Image 85. Common Wall, Pararge schakra (upper)

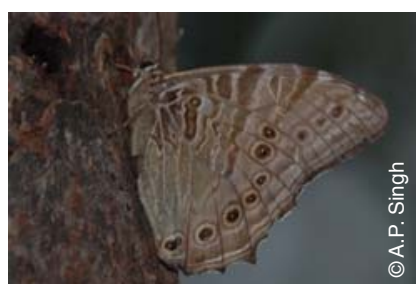

Image 89. Dusky Labyrinth, Neope yama (upper)

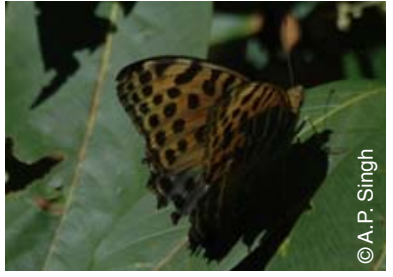

Image 70. Large Silverstripe, Childrena childreni (upper)

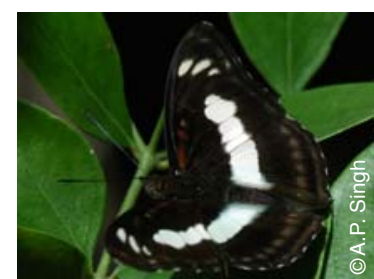

Image 74. Staff Sergeant, Athyma cama (upper)

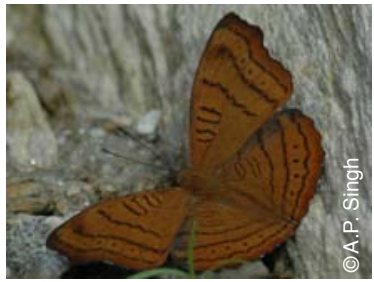

Image 78. Tabby, Psuedergolis wedah (upper)

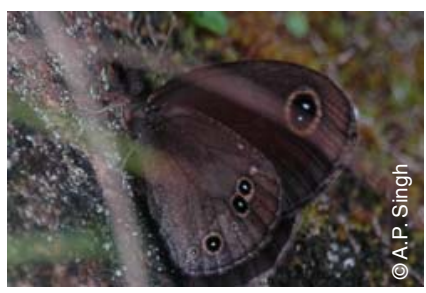

Image 82. Common Argus, Callerebia nirmala (under)

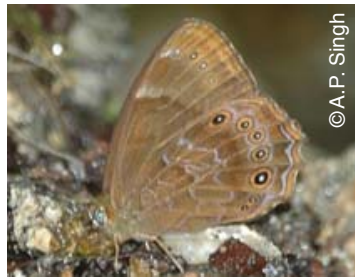

Image 86. Common Wood Brown, Lethe sidonis (under)

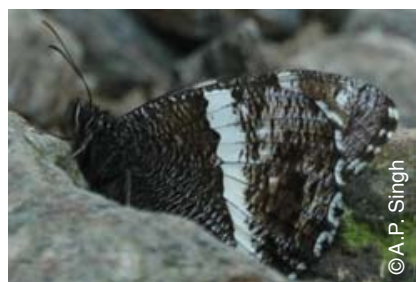

Image 90. Great Satyr, Aulocera padma (under) 


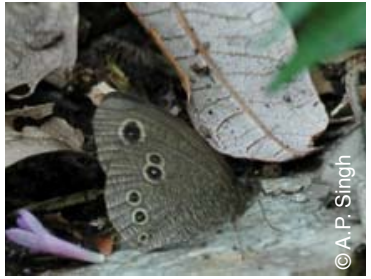

Image 91. Himalayan Five Ring, Ypthima sakra

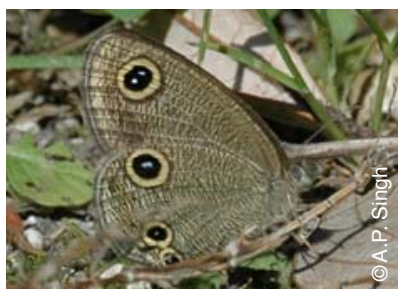

Image 95. Large Three Ring, Ypthima nareda (under)

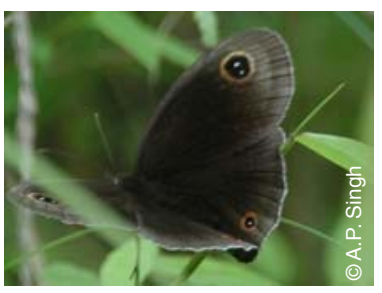

Image 99. Pallied Argus, Callerebia scanda (under)

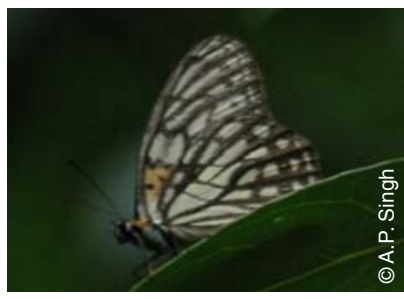

Image 103. TigerBrown, Orinoma damaris (upper)

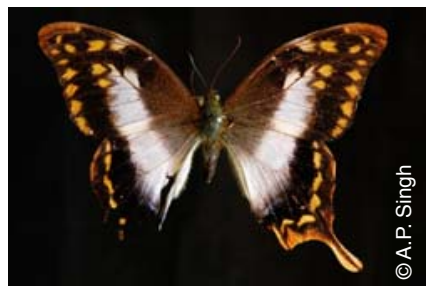

Image 107. Brown Gorgon, Meandrusa lachinus female

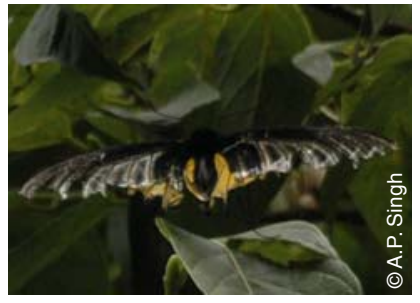

Image 111. Golden Birdwing,

Troides aeacus

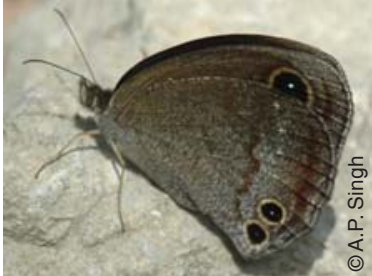

Image 92. Hybrid Argus, Callerebia hybrida (under)

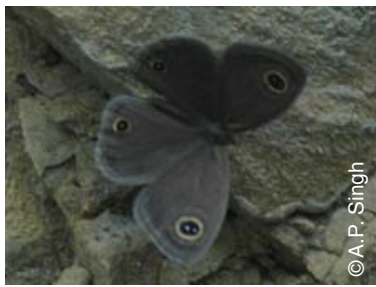

Image 96. Large Three Ring, Ypthima nareda (upper)

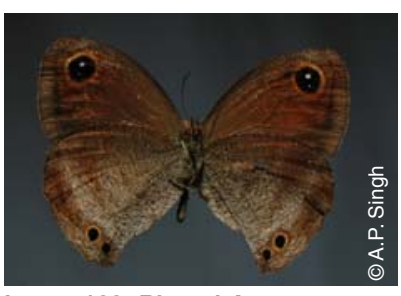

Image 100. Ringed Argus, Callerebia annada (under)

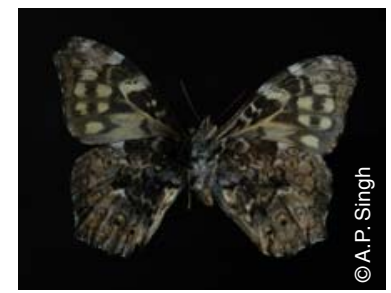

Image 104. Viened Labyrinth, Neope pulaha (under)

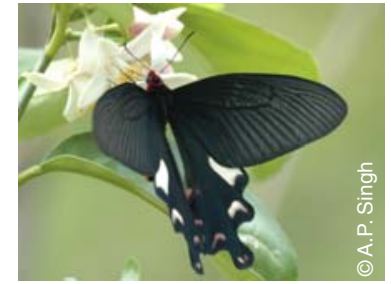

Image 108. Great Windmill, Atrophaneura dasarada

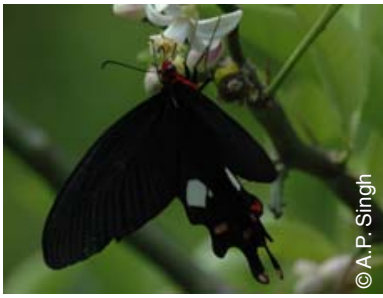

Image 112. Common Windmill, Atrophaneura polyeuctes

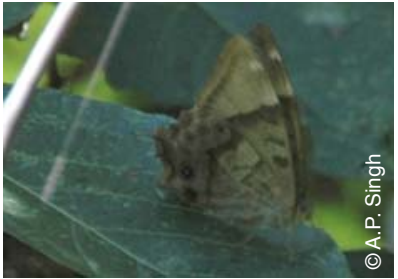

Image 93. Large Goldenfork, Zophoessa goalpara (under)

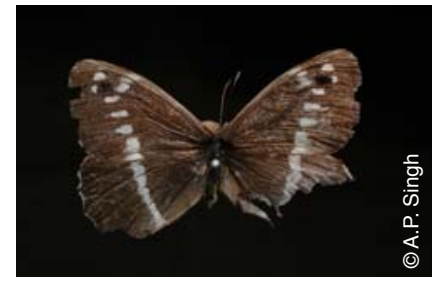

Image 97. Narrow-banded Satyr, Aulocera brahminus (under)

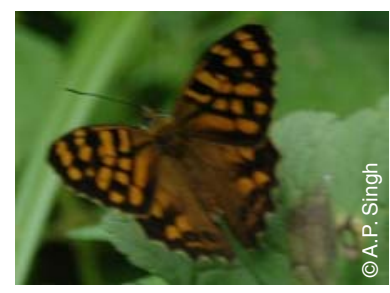

Image 101. Small Tawny Wall, Raphicera moorei (upper)

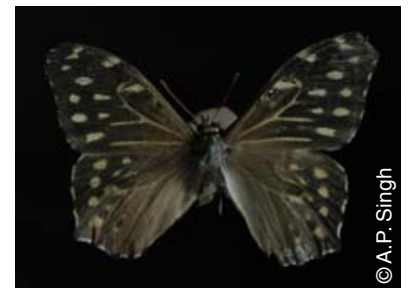

Image 105. Viened Labyrinth, Neope pulaha (upper)

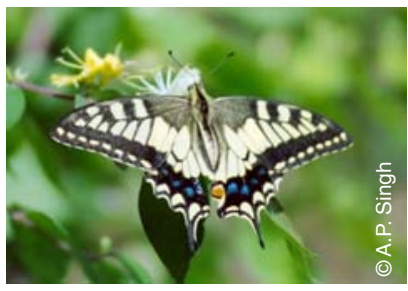

Image 109. Common Yellow Swallowtail, Papilio machaon

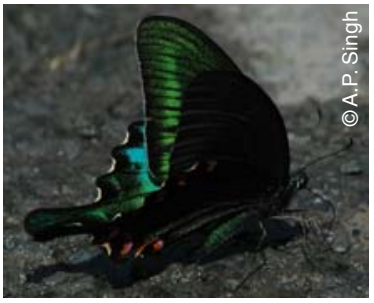

Image 113. Common Peacock Papilio bianor polyctor

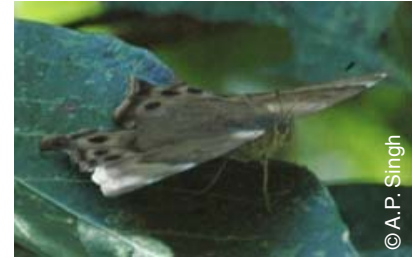

Image 94. Large Goldenfork, Zophoessa goalpara (upper)

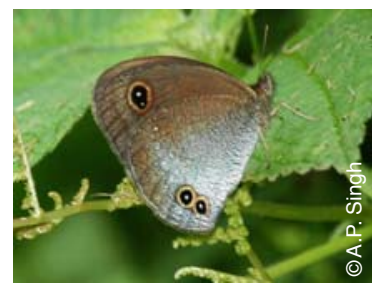

Image 98. Pallied Argus, Callerebia scanda (under)

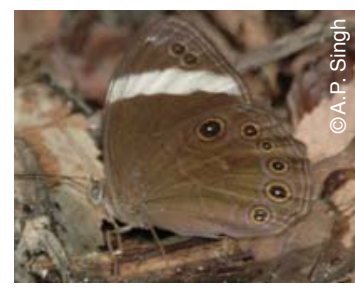

Image 102. Straight-banded Tree Brown, Lethe verma (under)

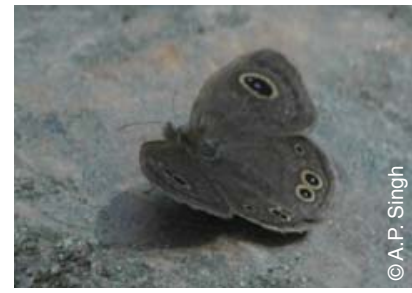

Image 106. Western Five Ring Ypthima indecorum (upper)

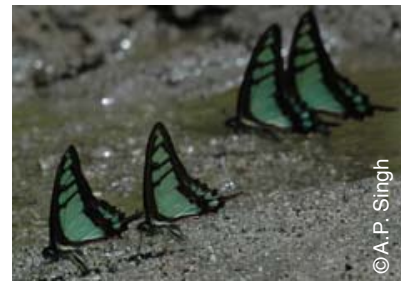

Image 110. Glassy Blue Bottle, Graphium cloanthus

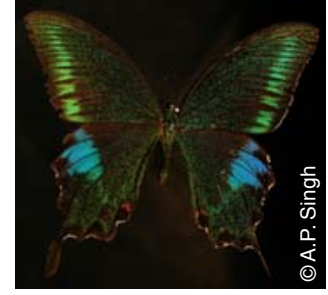

Image 114. Common Peacock Papilio bianor polyctor 


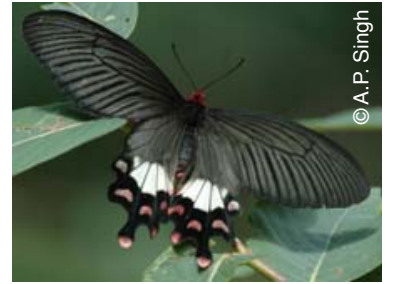

Image 115. Rose Windmill, Atrophaneura latreillei

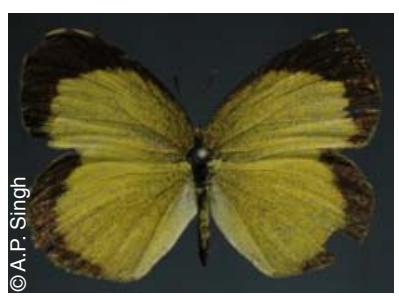

Image 119. Small Grass Yellow, Eurema brigitta (upper)

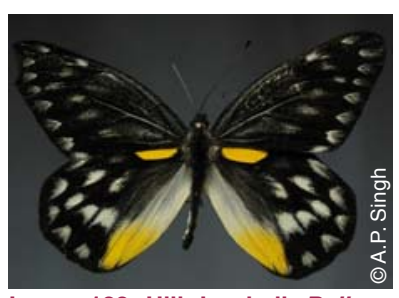

Image 123. Hill Jezebell, Delias belladonna (upper)

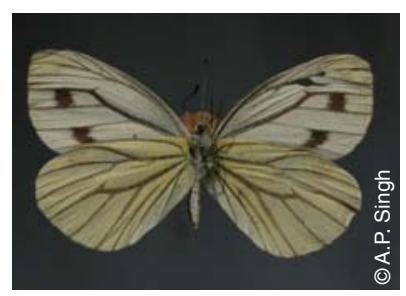

Image 127. Mountain Green-veined White, Pieris montana (under)

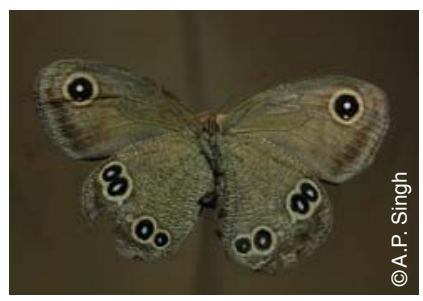

Image 131. Brown Argus, Dallacha hyagriva (under)

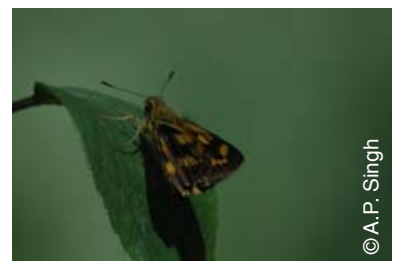

Image 135. Indian Dart, Potanthus pseudomaesa clio (upper)

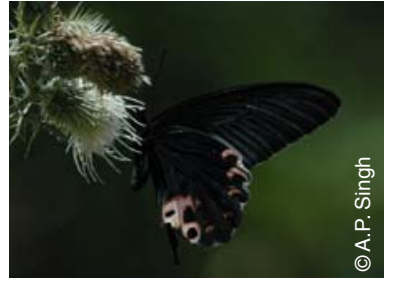

Image 116. Spangle, Papilio protenor

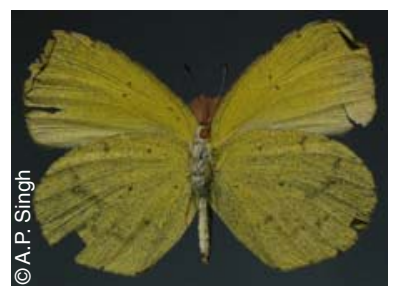

Image 120. Small Grass Yellow, Eurema brigitta (under)

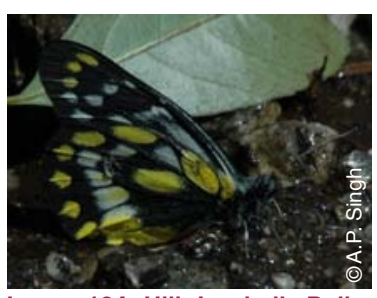

Image 124. Hill Jezebell, Delias belladonna

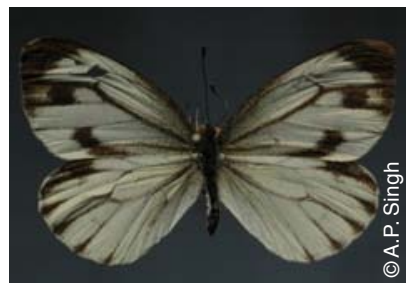

Image 128. Mountain Green-veined White, Pieris montana (upper)

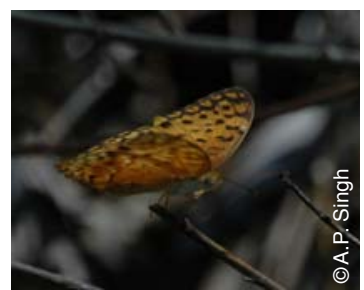

Image 132. Common Leopard, Phalantha phalantha

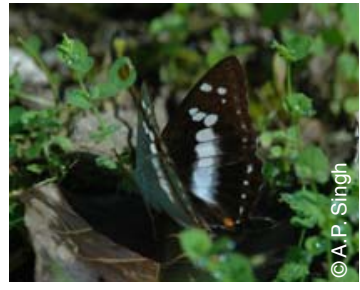

Image 136. Purple Emperor, Apatura ambica - female (upper)

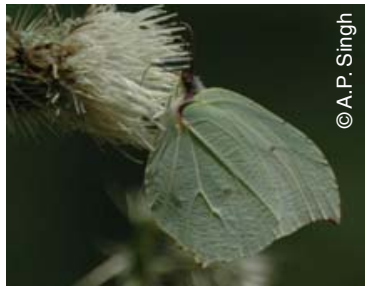

117. Common Brimstone, Gonepteryx rhamni

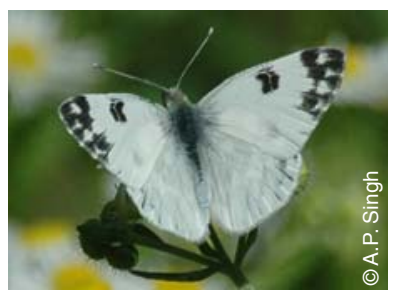

Image 121. Bath White, Pieris daplidice

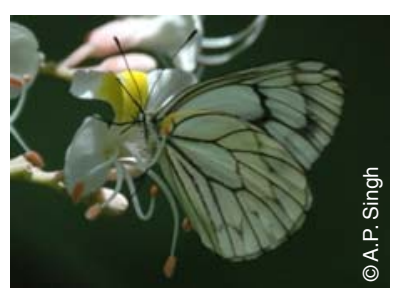

Image 125. Himalayan Black Vein Aporia leucodice

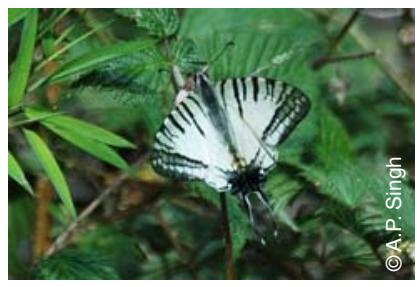

Image 129. Sixbar Swordtail, Pazala eurous (upper)

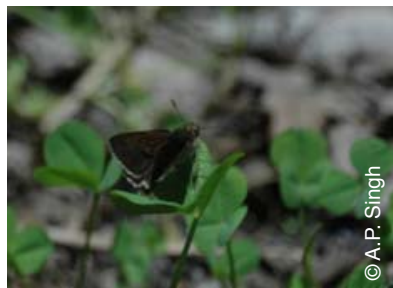

Image 133. Dingy Scrub Hopper, Aeromachus dubius (upper)

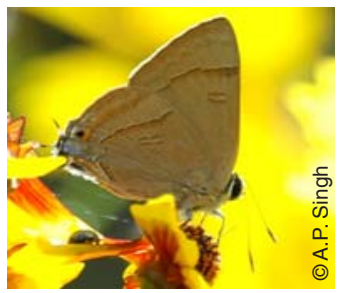

Image 137. Red Himalayan Flash, Rapala selira (under)

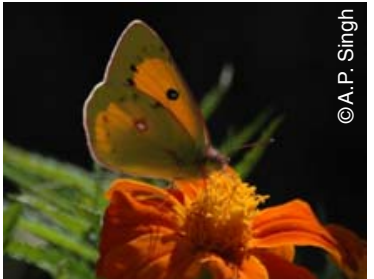

118. Dark Clouded Yellow, Colias fieldi

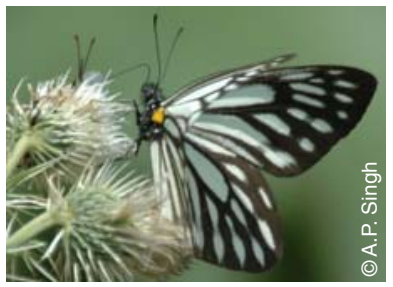

Image 122. Great Black Vein, Aporia agathon

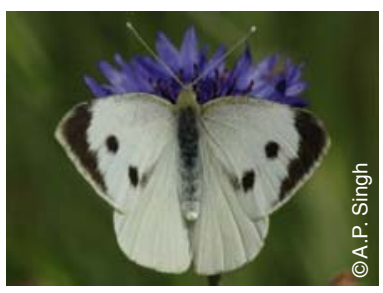

Image 126. Large Cabbage White Pieris brassicae

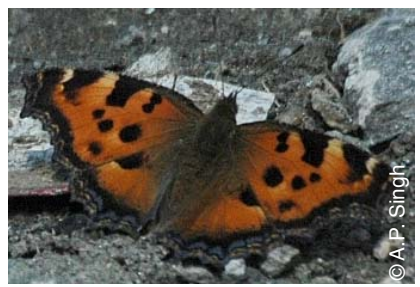

Image 130. Large Tortoiseshell, Nymphalis xanthomelas (upper)

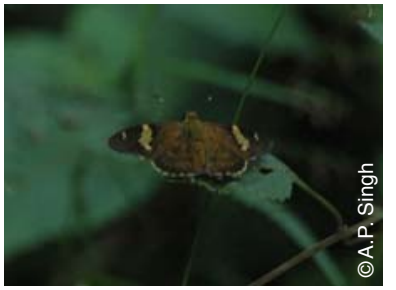

Image 134. Himalayan Yellow Flat, Celaenorrhinus dhanada (upper)

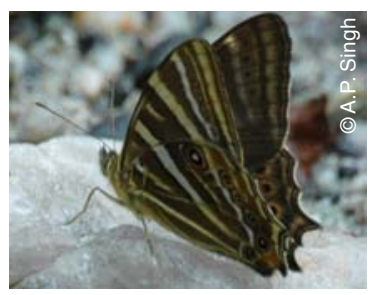

Image 138. Treble Silverstripe, Lethe baldeva aisa (under) 


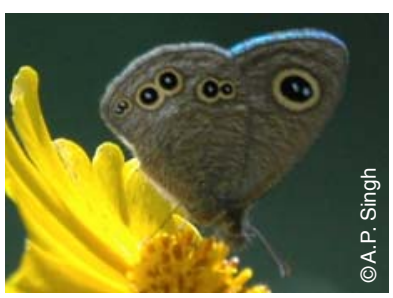

Image 139. Common Five Ring, Ypthima baldus (under)

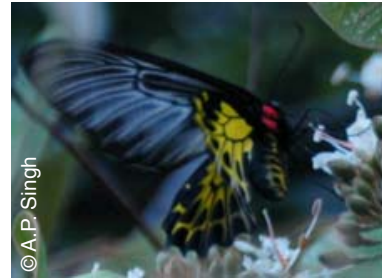

Image 140. Golden Birdwing, Troides aeacus female (under)

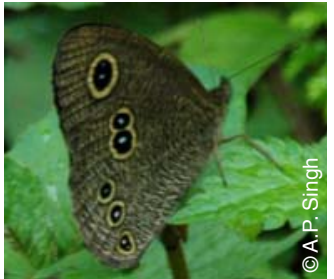

Image 141. Himalayan Five Ring, Ypthima nikaea (under)

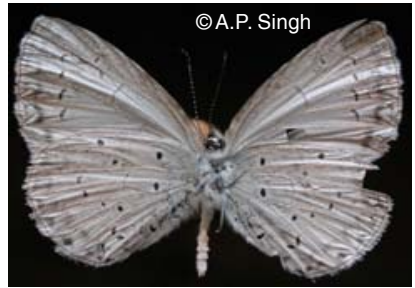

Image 142. Large Hedge Blue, Celasrina huegelii (under)

Singh, A.P. \& R.S. Bhandari (2003). Butterfly diversity in tropical moist deciduous sal (Shorea robusta) forests of Dehradun valley: the lower western Himalayas. Indian Forester 129: 1257-1269.

Singh, A.P. \& R. Pandey (2004). A model for estimating butterfly species richness of areas across the Indian sub-continent: species proportion of family Papilionidae as an indicator. Journal of the Bombay Natural History Society 101: 79-89.

Singh, A.P. (2006). Range extension of Brown Gorgon butterfly, Meandrusa gyas gyas into Kedarnath Musk Deer Reserve, Western Himalayas: a lesser known species from North-East India. Indian Forester 132(12): 187-189.

Singh, A.P. \& R. Singh (2007). Distribution, habitat and voice of Whitetailed Robin Myiomela leucura in Kedarnath Musk Deer Reserve, Garhwal Himalaya, India. BirdingASIA 8:66-67.

Singh, G., H.B. Naithani \& G.S. Rawat (2009). Observations on the flora of Mandal forest, Garhwal Himalaya. Indian Forester 135(2): in press.

Smith, C. (1989). Butterflies of Nepal (Central Himalayas). Craftman Press, Bangkok, 352pp.

Swinhoe, C. (1905-1910). Lepidoptera Indica Part VII. RhopaloceraPapilionidae \& Lycaenidae. Lovell, Reeve \& Co.Ltd, London, 286pp + 551-639pp.

Swinhoe, C. (1910-1911). Lepidoptera Indica Part VIII. RhopaloceraLycaenidae. Lovell, Reeve \& Co. Ltd., London, 293pp + 640-705pls.

Swinhoe, C. (1911-1912). Lepidoptera Indica Part IX. RhopaloceraLycaenidae \& Hesperiidae. Lovell, Reeve \& Co. Ltd., London, 278pp + 706-756pls.

Swinhoe, C. (1912-1913). Lepidoptera Indica Pars X. RhopaloceraHesperiidae. Lovell, Reeve \& Co. Ltd., London, 364pp + 757-835pls.

Talbot, G. (1939). The Fauna of British India including Ceylon and Burma - Butterflies. 2nd edition Vol-I. Taylor \& Francis, London, 600pp.

Talbot, G. (1947). The Fauna of British India including Ceylon and Burma - Butterflies. 2nd edition Vol-II. Taylor \& Francis, London, 506pp.

Uniyal, V.P. (2004). Butterflies of Nanda Devi National Park- a world heritage site. Indian Forester 130: 800-803.

Varshney, R.K. (1997). Index Rhopalocera Indica Part III. Genera of butterflies from India and neighbouring countries (Lepidoptera: (C) Lycaenidae). Oriental Insects 31: 83-138.

Walpole, M.L. \& I.R. Sheldon (1999). Sampling butterflies in a tropical rain forest: an evaluation of transect walk method. Biological Conservation 87: 85-91.

Wynter-Blyth, M.A. (1957). Butterflies of the Indian Region. Bombay Natural History Society, Bombay, 523pp. 